\title{
Identification and Analysis of Full Scale Ventilation Events
}

\author{
Luca Savio $^{1,2}$ and Sverre Steen ${ }^{1}$ \\ ${ }^{1}$ Department of Marine Technology, Norwegian University of Science and Technology (NTNU), \\ Otto Nielsens vei 10, N-7491 Trondheim, Norway \\ ${ }^{2}$ Department of Ship Technology, Norwegian Marine Technology Research Institute (MARINTEK), \\ Otto Nielsens vei 10, N-7450 Trondheim, Norway
}

Correspondence should be addressed to Luca Savio, luca.savio@marintek.sintef.no

Received 7 February 2012; Accepted 12 May 2012

Academic Editor: Yin Lu (Julie) Young

Copyright (C) 2012 L. Savio and S. Steen. This is an open access article distributed under the Creative Commons Attribution License, which permits unrestricted use, distribution, and reproduction in any medium, provided the original work is properly cited.

\begin{abstract}
The present paper deals with propeller ventilation in full scale. The paper is based on full scale monitoring data from an offshore supply ship during normal operation. The data was collected by the on-line monitoring system HeMoS, developed by Rolls Royce Marine. The data covering one year and a half of ship operations were made available within the framework of the Era-Net Martec project PropSeas. The ventilation events are identified by means of an analysis procedure based on fuzzy logic. The paper contains both a basic introduction to fuzzy logic and a detailed description of the analysis procedure. The analysis procedure is then adopted to process the available data, find ventilation events, and form a set which is further analyzed including weather observations.
\end{abstract}

\section{Introduction}

Propeller ventilation is the process of a ship propeller drawing air from the free surface while working. It is known that ventilation can lead to propeller racing and possibly to large loads on the blades and on the propeller drive shaft. Both of the mentioned effects of ventilation can cause failure of the propulsive system. For this reason ventilation has been intensively studied throughout the years by means of different methods.

Pioneer studies have been carried out by Kempf [1], Shiba [2], Gutsche [3], and Fleischer [4] analysing the effect of both the propeller geometry and the operational conditions on the ventilation phenomenon. However, these works were not taking fully into account the environmental condition the propeller is operating in, since the propeller was considered to have a static submergence. A more dynamic scenario has been reproduced by Koushan [5] and Koushan et al. [6] where the propeller was subjected to forced sinusoidal heave motion from completely submerged to completely out of water and to regular sinusoidal waves, respectively. The problem has been tackled also by means of CFD as presented in Califano [7] where he points out that also the compressibility of air, which is negligible in model scale, might influence ventilation in full scale. Finally the different ventilation types and inception mechanisms have been classified by Kozlowska et al. [8]. All these studies rely on some kind of approximations which aim to simplify the problem, so that it is possible to classify them under the category of ventilation in a controlled scenario. This kind of studies is important to understand the mechanism beyond ventilation and its effects, but does not take fully into account its link with the ship system. On the contrary the relation of ventilation with the ship system is the basis of this paper. The occurrence of ventilation is detected through its effects on the propulsive system of a ship during its normal operations.

This paper uses data provided by the Health and Monitoring System (HeMoS hereafter) which is currently under development by Rolls Royce Marine. The HeMoS system is an in-service monitoring system aiming to early assess possible failure of propulsive system components. The system is installed on a supply vessel, which is equipped with two pulling azimuthing thrusters for the propulsion. The system monitors many different subsystems, but in the present analysis only the two main propulsion units are considered. The system continuously logs at a sampling rate of $0.5 \mathrm{~Hz}$, the propulsive system status, and the ship dynamic positioning system at $1 \mathrm{~Hz}$. Such an advanced system has 
many possible benefits, among which is the possibility of helping the ship owner to better manage the ship. It was also found in an analysis carried out by Rolls Royce Marine that ventilation events can be detected. However the proposed procedure, though being effective, was mainly manual and hence not easily applicable to large amounts of data. In the present paper the method proposed by Rolls Royce Marine is extended and made suitable to analysis of long time-series by means of fuzzy logic. Moreover the data from almost one year and a half of ship operation are analyzed and compared with meteorological observations for the stretch of sea the ship is operating in.

The paper is divided into three sections. The first section includes a simple introduction to fuzzy logic and the development of the fuzzy logic toolbox. In the second the in-service data are analyzed. Finally in the last section the analysis is extended including the weather observations.

The data are made available by Rolls Royce Marine within the framework of the cooperative research project PropSeas.

\section{The Analysis Toolbox}

2.1. Concepts behind the Analysis Toolbox. The data analysis toolbox for the full scale recordings was necessary due to the huge amount of data to be analyzed. The data collected by the onboard acquisition system HeMoS are of two types: the propulsive system data and the ship environmental data from the dynamic positioning system. The sampling frequencies are $0.5 \mathrm{~Hz}$ and $1 \mathrm{~Hz}$, respectively. Though the sampling frequencies are rather low, the time span covered by the acquisition is quite large, leading to long time series to be analyzed. This huge amount of data means that if the data had to be analyzed by a human, the work would have been long and tedious. Moreover the analyses performed by humans are subjected to the arbitrary interpretation of the single human looking at the data. However the human thinking, and its capability to easily incorporate new input and criteria, is necessary in such an analysis. It was necessary, therefore, to create a framework in which the human could analyze data, formulate hypotheses, and check if the hypotheses were correct in a rigorous, but at the same time flexible way. A solution to this twofold necessity was found to be fuzzy logic.

It has also to be remarked that the low frequency sampling does not allow for adopting other common time series analysis techniques. Early attempt to adopt cross correlation between signals or pattern recognition techniques proved to be unsuccessful due to the very low sampling frequency.

2.2. Crisp versus Fuzzy Logic. Fuzzy logic or more precisely fuzzy sets were first introduced by Zadeh in 1965 [9]. From that time on fuzzy logic has been increasingly applied to different fields which include control, decision making, and approximate reasoning. Although fuzzy logic can be presented in a formal way, we prefer here to introduce it through an example. For a formal introduction to fuzzy logic and approximate reasoning the reader can refer to Zadeh [10]. The background idea of fuzzy logic is that in the human way of classifying things there are many sets which have a blurry boundary. For this reason when introducing fuzzy logic, it is useful to compare it with crisp logic, which is the one we are all familiar with.

The main difference between crisp and fuzzy logic is that the first one assigns a binary number $[0,1]$ to the truth value (value indicating the relation of a proposition to truth), while fuzzy assigns a truth value which is a real number that ranges from 0 to 1 . This blurry definition of the truth value is what makes fuzzy logic suitable for representing the human thinking in mathematical terms. We humans are used to express concepts in a smooth way. If we consider, for instance, air temperature in a region where it might span from 0 to $30^{\circ}$ degrees and today is 14 degrees, then the question is to decide if today is hot or cold. The crisp logic approach would be to set a threshold at 15 degrees, and since today the temperature is 1 degree below the threshold state, then today is cold. But if the temperature was 16 degrees then today would have been hot. Probably most of us would have said that today is a warm day. However in a two-value logic this is not possible. Fuzzy logic on the contrary assigns a membership to today's temperature, which is to what degree a sample belong to a set (in this example how much today's temperature belongs to the set of hot days). So if the temperature range from 0 to 30 degrees is linearly spaced, then the membership of today's temperature to the set of the hot days is $14 / 30=0.467$. However this might still be considered to be somewhat rough. Humans do not feel the temperature on a linear scale, thus the linear mapping from temperature to membership should be changed in favor of a more suitable one. This mapping between a sample and its membership $(\mu)$ to a given set is carried out through the so-called membership function. Selecting a suitable membership function is crucial in fuzzy logic. The membership function is an arbitrary mathematical expression which maps from measured value to membership. Although the function is arbitrary, there is a set of functions which have been extensively used in fuzzy logic. In this case a suitable one might be the sigmoid right, which has the following expression:

$$
\mu=\frac{1}{\left(1+e^{\alpha \cdot(x-\beta)}\right)},
$$

where $\mu$ denotes the membership, $x$ a parameter of the sample under examination, $\alpha$ controls the steepness of the curve and $\beta$ the center of the curve (in the sense that a membership equal to 0.5 is given to a sample whose $x$ equals $\beta$ ). The parameter $\alpha$ acts as a gain while the $\beta$ as an offset, since the first controls the steepness of the curve whereas the second is where the curve is centered.

The sigmoid right has the general shape (a smooth step) shown in Figure 1.

The sigmoid right can be tuned for the temperature example so that a membership equal to 0.5 is assigned to 15 degrees and that below 5 degrees is cold and above 25 degrees is hot by choosing $\beta$ equal to 15 and $\alpha$ equal to -0.5 . The crisp logic and the two fuzzy approaches are compared in Figure 2. 


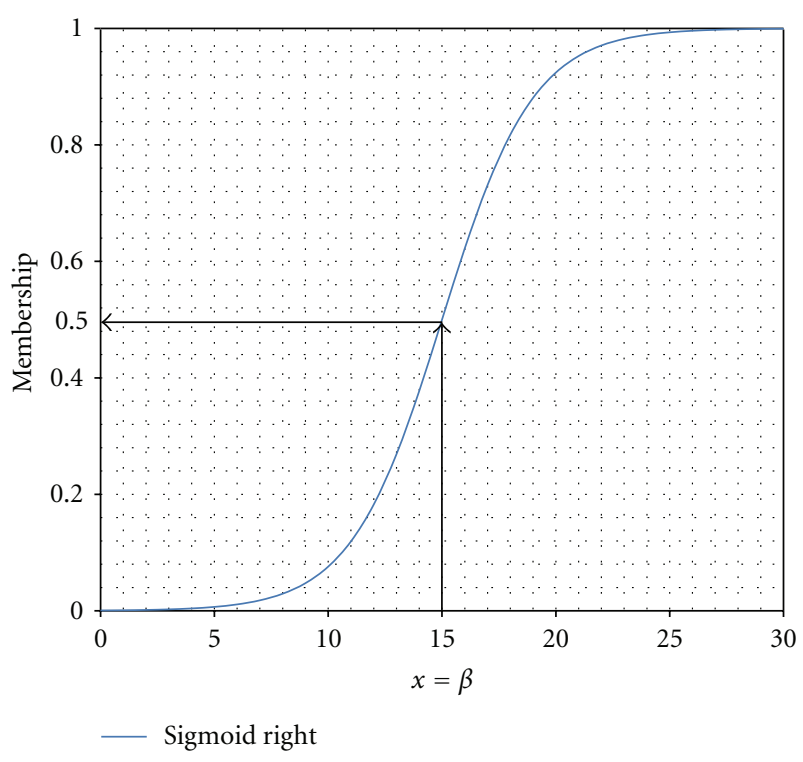

FIGURE 1: Sigmoid right membership function.

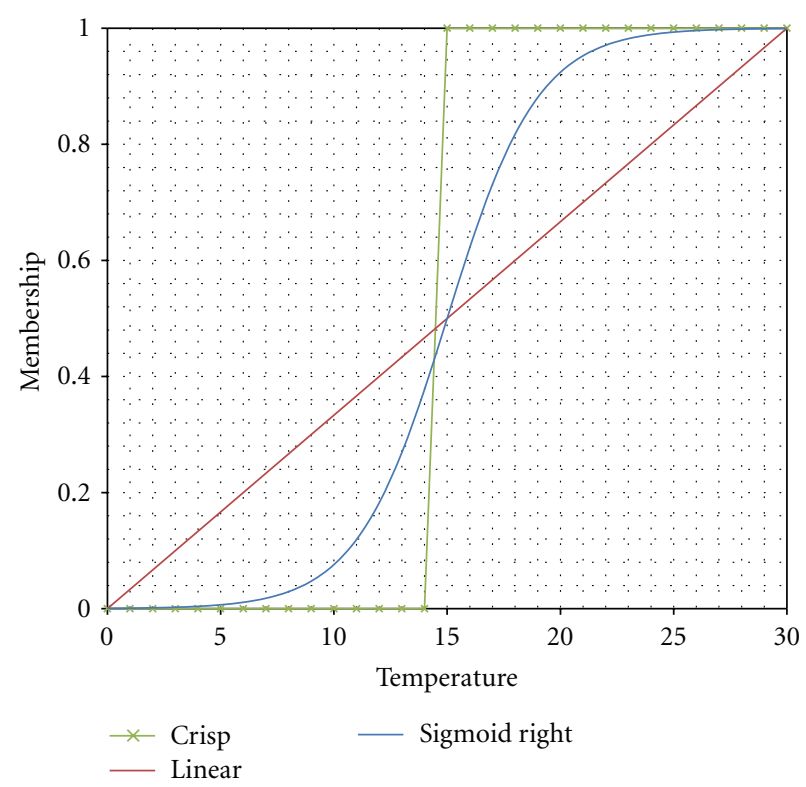

FIGURE 2: Comparison of membership functions: green-crisp logic approach, red-fuzzy linear, and blue-fuzzy sigmoid right.

What has been presented above is just the basic tool of the fuzzy logic: the membership function. The great advantage of fuzzy logic comes when more than one criterion is used to make a decision. If one wants to add additional criteria in order to establish if today is hot or not, this can be handled easily by fuzzy logic. For example, one might think to add information related to air humidity, wind speed, cloud cover creating new membership functions, suitable for representing how the newly added parameter is mapped to the set of hot days (for instance high humidity increases the chances for today to be hot). Dealing with many criteria with crisp logic results generally in very complicated systems which have to consider every single combination of the parameters taken into account in order to get to a conclusion. The fuzzy logic approach on the contrary is to define criteria and then express them through membership functions. However, calculating the membership functions does not lead directly to a final conclusion. Once all the memberships of a given sample have been calculated, the so-called fuzzy set is formed. The next step, which corresponds to finally assign the value 1 or 0 to a given sample, is called defuzzy. Although the de-fuzzy step can be carried out in many different ways, here just one approach is presented. In this approach a weight is given to each of the membership functions and then a threshold is set to the sum of the membership functions. Samples below the threshold are left outside the set. When fuzzy logic is applied the way that has been described, it is common to refer to the procedure as fuzzy inference. Therefore the developed toolbox belongs to the category of fuzzy logic inference systems.

2.3. Available Data from the Propulsive and the Dynamic Positioning Systems. As already mentioned this analysis is based on a set of data from two different systems installed onboard, the propulsive apparatus monitoring and the dynamic positioning systems. The HeMoS system is a monitoring system of the health of the entire propulsive system and just a subset of the collected data refers to the main propulsion system. The list of all the channels available for this analysis is reported in Table 1 .

Along with the above-listed channels, some derived channels are calculated with the aim of making the analysis simpler. In particular the usual torque $K_{Q}$ and advance $J$ coefficients have been introduced:

$$
\begin{gathered}
K_{\mathrm{Q}}=\frac{Q}{\rho n^{2} D^{5}}, \\
J=\frac{V}{n D},
\end{gathered}
$$

where $Q$ is propeller torque, $\rho$ the water density, $D$ the propeller diameter, $n$ the propeller number of revolutions, and $V$ the vessel speed. The convention for the azimuth angle is that it is positive when the unit is rotated in clockwise direction, when the unit is seen from above. When the port unit has a positive azimuth, the port propeller is closer to the ship center line (we refer to this condition as inward). The starboard propeller is closer to the ship center line when the starboard unit azimuth angle is negative. The convention for the pitch angle is that it is positive when the bow moves up.

In addition to the standard propeller coefficient and in consideration of the fact that ventilation is involved in the analysis, a parameter representing the propeller submergence has been included. It is not possible from the data available to calculate the propeller submergence exactly, since no information is available for the actual wave elevation at the propeller location. However a parameter linked to the static submergence, due to the instantaneous ship roll and pitch angle, was deemed important. It is important to remark that in a fuzzy logic approach it is not necessary to precisely calculate the static submergence, and it is sufficient to define 
TABLE 1: List of available channels.

\begin{tabular}{|c|c|c|}
\hline System & Channel name & Notes \\
\hline Propulsive & Azimuth feedback & Thruster azimuth angle, positive clockwise seen from above \\
\hline Propulsive & Load feedback & Not used by the toolbox \\
\hline Propulsive & Pitch feedback & $\begin{array}{l}\text { Propeller pitch as a percentage of the maximum pitch (which depends on the ship } \\
\text { operating mode) }\end{array}$ \\
\hline Propulsive & Rpm feedback & $\begin{array}{l}\text { Propeller speed as a percentage of the maximum speed (which depends on the ship } \\
\text { operating mode) }\end{array}$ \\
\hline Propulsive & $\begin{array}{l}\text { Torque feedback } \\
\text { Drive of motor }\end{array}$ & Propeller torque as a percentage of the maximum torque \\
\hline Propulsive & Vessel speed & \\
\hline Dynamic positioning & Latitude & Not directly used by the toolbox \\
\hline Dynamic positioning & Longitude & Not directly used by the toolbox \\
\hline Dynamic positioning & GPS speed & Not used by the toolbox \\
\hline Dynamic positioning & Heading of the ship & Not directly used in the toolbox \\
\hline Dynamic positioning & Ship pitch & Used in the submergence index \\
\hline Dynamic positioning & Ship roll & Used in the submergence index \\
\hline Dynamic positioning & Wind direction & Not used \\
\hline Dynamic positioning & Wind speed & Not used \\
\hline
\end{tabular}

an index which is linked to it. Therefore the submergence index was defined as follows:

$$
\text { submergence_index }=-3^{*} \tan (\text { pitch })+\operatorname{sign}{ }^{*} \tan (\text { roll }) \text {, }
$$

where pitch and roll are ship pitch and roll angles, respectively, and sign is equal to one if the port side unit is considered, otherwise it is -1 . A More accurate estimate of the static submergence can be obtained by substituting the weights 3 and 1 to the pitch and roll motions, respectively, by the actual position of the propeller centers with respect to the center of gravity and using the nonlinear Euler rotation. Unfortunately, when the toolbox was developed, neither the exact position of the propellers nor the center of gravity of the ship was known.

2.4. Development of the Fuzzy Logic Toolbox. The first step in developing a fuzzy inference system is to express criteria in linguistic terms. This step, with reference to the temperature example, would correspond to state that "if today is a hot day, then the temperature must be high." In the case of the HeMoS system for each sample more than one parameter is available, so more than one criterion can be defined. The criteria used for the present analysis are the following.

(1) If propeller revolutions show a sudden increase that is probably due to ventilation.

(2) If the torque (or more precisely $K_{Q}$ ) shows a sudden drop, this is likely an interesting event.

(3) If the submergence index is high the propeller is likely to be close to the free surface, increasing the chances of ventilation to occur.

(4) On the contrary if the propeller pitch is suddenly reduced, the corresponding $K_{Q}$ reduction might not be caused by ventilation.
(5) If the thruster is pointing inwards (azimuth angle), the hull shelters the propeller and hence ventilation is less probable.

These criteria have been defined based on the thruster designers experience and the observation of the data collected so far. The first two criteria are also supported by what is reported to be consequences of ventilation on the ship propulsive system by other authors. For a summary of the effects of ventilation on the propulsion of the ships the reader can refer to Smogeli and Sørensen [11]. The third criterion is meant to add a rough estimate of the likelihood that the propeller is close enough to the free surface to draw air. The fourth criterion is related to the specific application presented here. The propeller is a controllable pitch propeller and hence the control system acts also on the propeller pitch. It was found that when the ship is subject to rough sea states, the control system acts from time to time on the propeller pitch, reducing it, probably to limit the torque. The pitch is decreased rapidly and this results in a sudden torque drop which can be easily misinterpreted as a ventilation event according to criterion 2. The fifth criterion is somewhat related to the third. The ship is equipped with two pulling azimuthing thrusters. The two units are installed underneath the ship stern and hence the air cannot be drawn from right above the propeller. When one of the units is rotated so that the propeller is closer to the ship center line, the path the air has to travel through to get to the propeller is longer, reducing the probability that ventilation develops.

It has to be pointed out that this set of criteria was selected in order to detect ventilation events during transit mode. For instance, as it will be shown later the dynamic positioning condition cannot be handled with these criteria.

In Table 2 the adopted membership functions are presented, after being loosely tuned on a first set of available data. It was chosen to loosely tune the membership function 
TABle 2: Membership function definition.

\begin{tabular}{lcc}
\hline Parameter & Membership function & Expression \\
\hline Propeller number of revolution $(\mathrm{rps})$ & Sigmoid right & $\mu_{n}=1 /\left(1+e^{-1.4 \cdot\left(n-\left(\bar{n}+\sigma_{n}\right)\right)}\right)$ \\
Torque coefficient $K_{Q}$ & Sigmoid left & $\mu_{K_{Q}}=1 /\left(1+e^{\left(0.8 / \sigma_{K_{Q}}\right) \cdot\left(K_{Q}-\left(\bar{K}_{Q}-2 \cdot \sigma_{K_{Q}}\right)\right)}\right)$ \\
Submergence index & Sigmoid right & $\mu_{\text {sub_idx }}=1 /\left(1+e^{-30 \cdot\left(\operatorname{sub} \_ \text {idx }-0.2\right)}\right)$ \\
Propeller pitch & Bell shaped & $\mu_{\text {pitch }}=1 /\left(1+|(p-\bar{p}) / 0.05|^{3}\right)$ \\
Azimuth & Sigmoid right or left & $\mu_{\mathrm{az}}=1 /\left(1+e^{\text {sign } \cdot 1.8 \cdot(\mathrm{az}-\mathrm{sign} \cdot 10)}\right)$ \\
\hline
\end{tabular}

in order not to focus too much on events like those used for the tuning. It has to be pointed out that the entire toolbox was developed and tuned having as goal to automatically recognize ventilation events, based on a set of events which have been already recognized by human operators.

The overbar denotes the median value of a variable taken over a sliding window of 61 samples ( $\approx 2$ minutes). The standard deviation $\sigma$ is calculated over the same window. The available data include only feedback signal and hence the median value is taken as representative of the value ordered by the control system.

The five membership functions can be divided into two groups by their nature. The propeller revolutions and the torque coefficient form the first group. Their aim is to detect outliers, in other words samples which do not fit with the statistics of the signal in the observation window. The standard deviation is therefore included in the relative membership function. Ideally the first group membership functions should output a membership value as close as possible to zero for most of the samples and assign a membership equal to one for the few outliers. The selected membership function is a sigmoid, which assigns a membership equal to 0.5 to a sample which makes the exponential argument equal to zero. In order to consider a sample of the propeller rps to be an outlier, that sample has to be higher than the mean value plus the standard deviation. For the torque coefficient the requirement is stricter. The torque coefficient has to be lower than the mean torque coefficient minus twice the relative standard deviation. To which degree a sample is then considered to be an outlier is controlled by the "gain factor" which is fixed for the propeller rps and a function of the torque coefficient standard deviation for the torque. The gain is fixed for the propeller rps since the signal shows rather constant statistical properties, whereas the gain is variable and related to the standard deviation for the torque coefficient since the signal varies significantly with the sea state.

The second group includes the pitch, the azimuth angle, and the submergence index membership functions and aims to check that the conditions are suitable for ventilation to happen. For the propeller pitch a different membership function was used due to the fact that the pitch feedback signal is rather different from the others. When the ship is not in a transient between two operating conditions, the pitch is kept constant by the control system and reduced just to avoid overloads. However due to the low resolution of the pitch feedback signal, the logged value of the pitch is found to jump back and forth between two levels during conditions where no change of pitch should happen. This effect is called quantization. Moreover when the control system acts, the reductions are rather limited. It was therefore necessary to select a membership function which is both not affected by the signal quantization and still capable of detecting rather small pitch changes. For this last signal the bell-shaped membership function was adopted. The general form of the bell-shaped function is

$$
\mu=\frac{1}{1+|(x-a) / c|^{2 b}} .
$$

The function is centred in $a$, where the membership for $x$ equal to $a$ is 1 . The function is flat about $a$ over a region which is controlled by the parameter $c$ and falls outside that region according to the parameter $b$. The flat region eliminates the problems related to the quantization of the signal. The curve can be made extremely steep outside the flat region acting on the $b$ parameter. The azimuth angle membership is calculated through a standard sigmoid function and it is tuned so that when the unit is pointing inwards for angles larger than 20 degrees, the membership is zero.

The submergence index represents a simple application of the sigmoid membership function. It was noticed that the ventilation events, which were already identified when the toolbox was developed, had a submergence index exceeding 0.2 with a rather high concentration around 0.25 . The submergence index sigmoid therefore has an offset equal to 0.2 and is rapidly increasing for indexes larger than 0.2 .

As described in the introduction to fuzzy logic, calculating the membership functions contributes to create the fuzzy set, which still needs to be defuzzified in order to get to the final conclusion that at a given time the propeller was ventilating. A simple way of defuzzifying is to set a threshold to the sum of the weighted membership functions above which a sample is considered to be ventilation. The choice of the weights reflects the relevance that is assigned to each criterion and the threshold level the degree of certainty required to include a sample in the set of the ventilation events. In this analysis the fuzzy set $F$, which is the sum of weighted membership functions, is calculated as follows:

$$
F=0.1 \mu_{n}+0.4 \mu_{K_{Q}}+0.15 \mu_{\text {sub_idx }}+0.15 \mu_{\text {pitch }}+0.2 \mu_{\text {az }} .
$$

The threshold has been set to 0.85 . If the threshold is set to 0.85 , then the weights can be divided into two groups. The first group includes parameters which are thought to be key factors to define a ventilation event and hence if their 


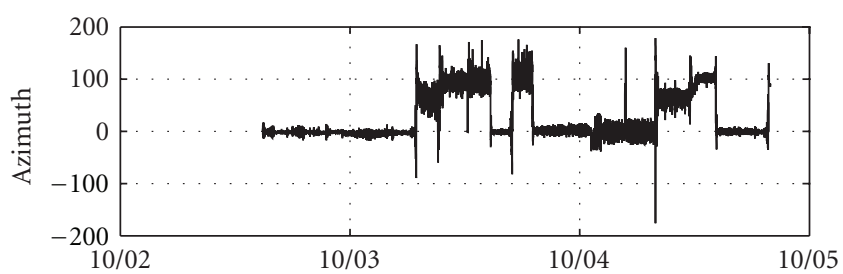

(a)

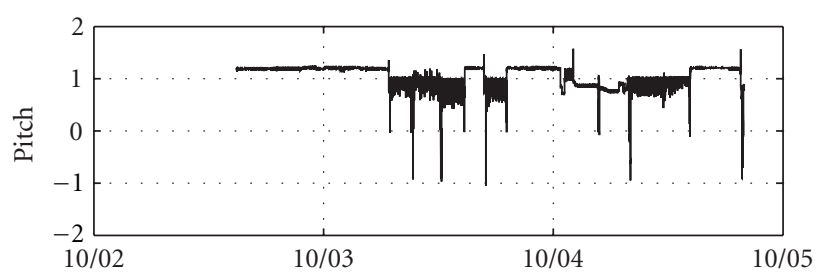

(c)

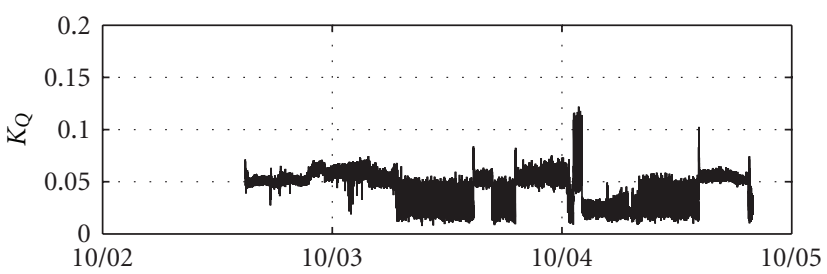

(e)

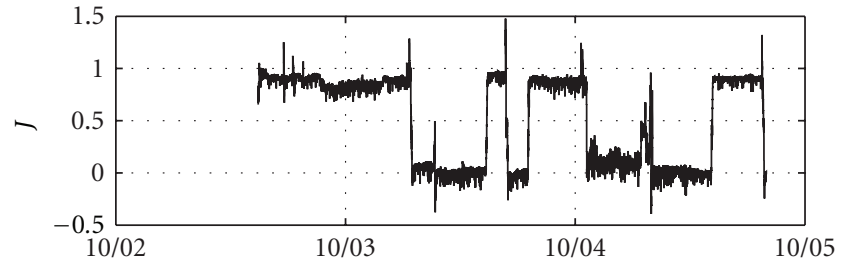

(b)

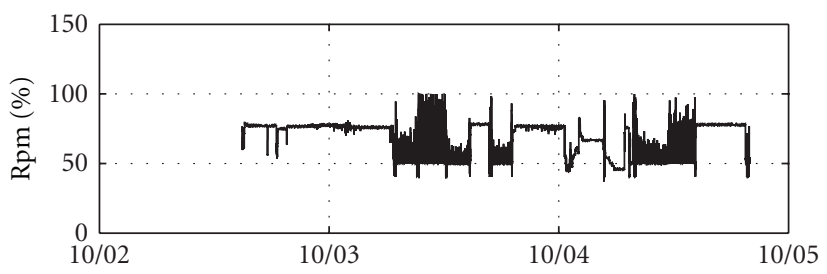

(d)

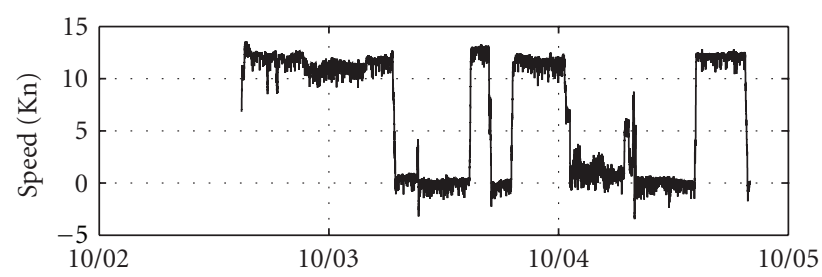

(f)

Figure 3: Time series of the training set.

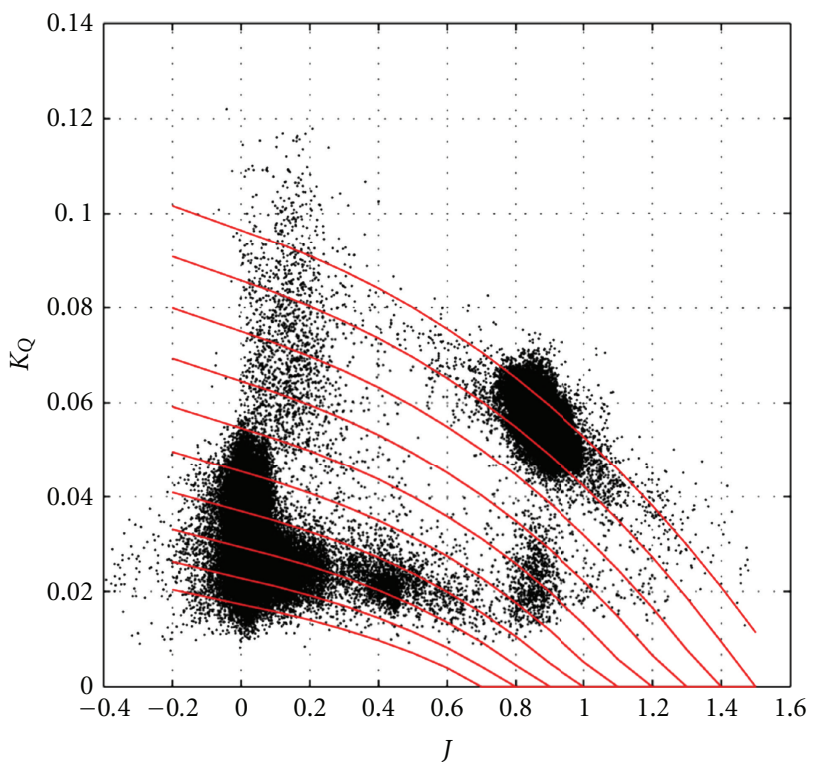

FIGURE 4: Torque coefficient against advance ratio.

membership is equal to zero, then that sample cannot be considered to be a ventilation event. To this group belong the torque coefficient and the azimuth angle. The torque coefficient lies in this group since the interest is on events which cause significant effects on the propulsive system. The azimuth angle belongs to this group because there are no clear evidences that any ventilation events happened when the units were pointing inwards. The second group includes the remaining three parameters; if their membership is zero, a sample can be still recognized as a ventilation event provided that the other memberships are equal to 1 . The lowest weight has been assigned to the propeller revolutions since it was noticed that not all the ventilation events caused a propeller racing event (probably the control system is preventing some of them). The submergence index cannot be considered to be a key factor of ventilation since the actual surface elevation is unknown. The same applies for the propeller pitch, since ventilation can also happen when the pitch has been already reduced by the control system.

It has to be remarked that the toolbox was tuned in order to avoid as much as false positives. The training set was too small to set up a detailed tuning procedure for the fuzzy logic parameters. It was instead decided to loosely tune the toolbox on a small training set, and then check on the available data if that was already a good tool to analyze long time series.

The application of the fuzzy toolbox will be shown first on the training set and then on another time series.

2.5. The Training Set. The toolbox was developed starting from an analysis performed on the HeMoS data by RollsRoyce Marine. The data used as the training set are reported in Figure 3 in the form of time series.

The time span over which the data have been acquired is around two days. Though the sampling frequency is rather low, the continuous logging led to a huge amount of data also in a rather short period of time as it is shown by plotting the torque coefficient against the advance coefficient. This plot is reported in Figure 4, where the red lines refer to the open 


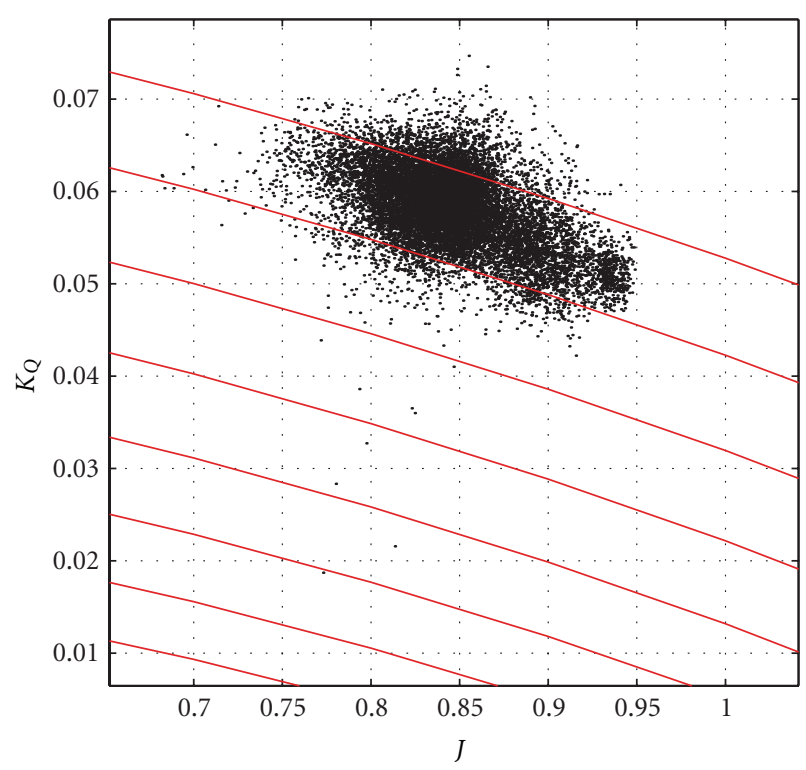

FIGURE 5: Points whose pitch was larger than $95 \%$ of the maximum pitch.

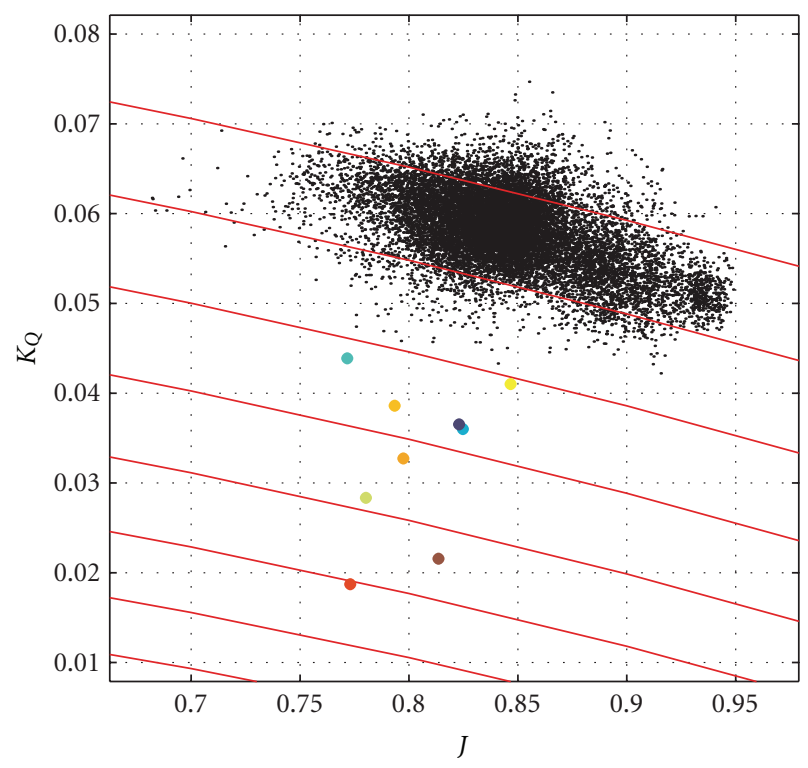

FIGURE 6: Flagged points.

water characteristics of a standard B-Series propeller of different pitch values, ranging from 0.5 to 1.4 . It is clear that the ship undergoes different operational conditions, which form the groups of points somewhat visible in the plot.

The strategy initially proposed in the analysis performed by Rolls Royce Marine was to identify a particular group of points representing an operational condition and then analyze the outliers. In the original analysis it was chosen to focus on points whose propeller pitch was larger than the $95 \%$ of the maximum one. The points that might relate to ventilation are those lying under the main group approximately at $J=0.8$ in Figure 5. However some of those points lying in that region might not be related to ventilation. It was then necessary to find in the time series other parameters confirming that those points were actually ventilation events, for example, large ship motions or sudden propeller rps increase. Although this is good, analysis strategy is impractical for long time series, since it requires a huge human effort.

At a first stage of the development of the toolbox these points which were flagged by this analysis were used to select and tune the membership functions. In Figure 6 the marked points are shown as colored dots, and then in Figure 7 presented also in the time series.

The calculated membership functions are presented in Figure 8.

The time histories of the five membership functions are shown in Figure 8 . The membership functions can be divided into two groups: the first group includes the first three whose aim is to mark outliers and the second group includes the remaining two whose aim is to eliminate false events. The goal for the first three would be to have a zero or very low membership for all the samples apart from those related to ventilation. On the contrary the last two check that the outlier samples possibly identified by the first three are not related to a sudden change in the blade pitch or that the propeller is sheltered by the hull. Based on the calculated membership function, it is possible to calculate the fuzzy set $F$, which is reported in Figure 9. The training set already offers two good examples of advantages and disadvantages of the fuzzy approach. If the threshold is set to 0.85 , all the flagged points would have been included apart from two. It is interesting to understand the difference between these two points circled in red and green. If the point circled in red in Figure 9 is considered, the toolbox would have correctly left it out, since the large torque drop was caused by a sudden reduction of the propeller pitch. Moreover the membership function relative to the submergence index for that point is zero. The probability that this point was related to ventilation is very low, as correctly predicted by the toolbox. The point marked in green is erroneously not included in the set of the ventilation events, because its fuzzy set value is rather low. However this event was probably a minor ventilation event which created rather limited effect on the propulsive system, which could have been included by selecting a lower threshold level. The chosen level of 0.85 is a rather conservative value and hence only major events can be detected.

It should also be noted that the toolbox is not capable of detecting events during dynamic positioning operation of the ship. Although this last point is clearly a limitation of the tool, it has to be noticed that there is clear evidence that ventilation is occurring during dynamic positioning operations. The limitation arises from the fact that the ordered values are not available. The control system changes propulsive parameter often during the 2 minutes time span used for approximating the ordered value, and hence the mean value, as above defined, has little to do with the actual ordered value. In Figure 10 an example of typical time series for the propulsive system, while the ship was in dynamic positioning condition, is presented. From the figure it is clear that the control system acts continuously on the system to keep 

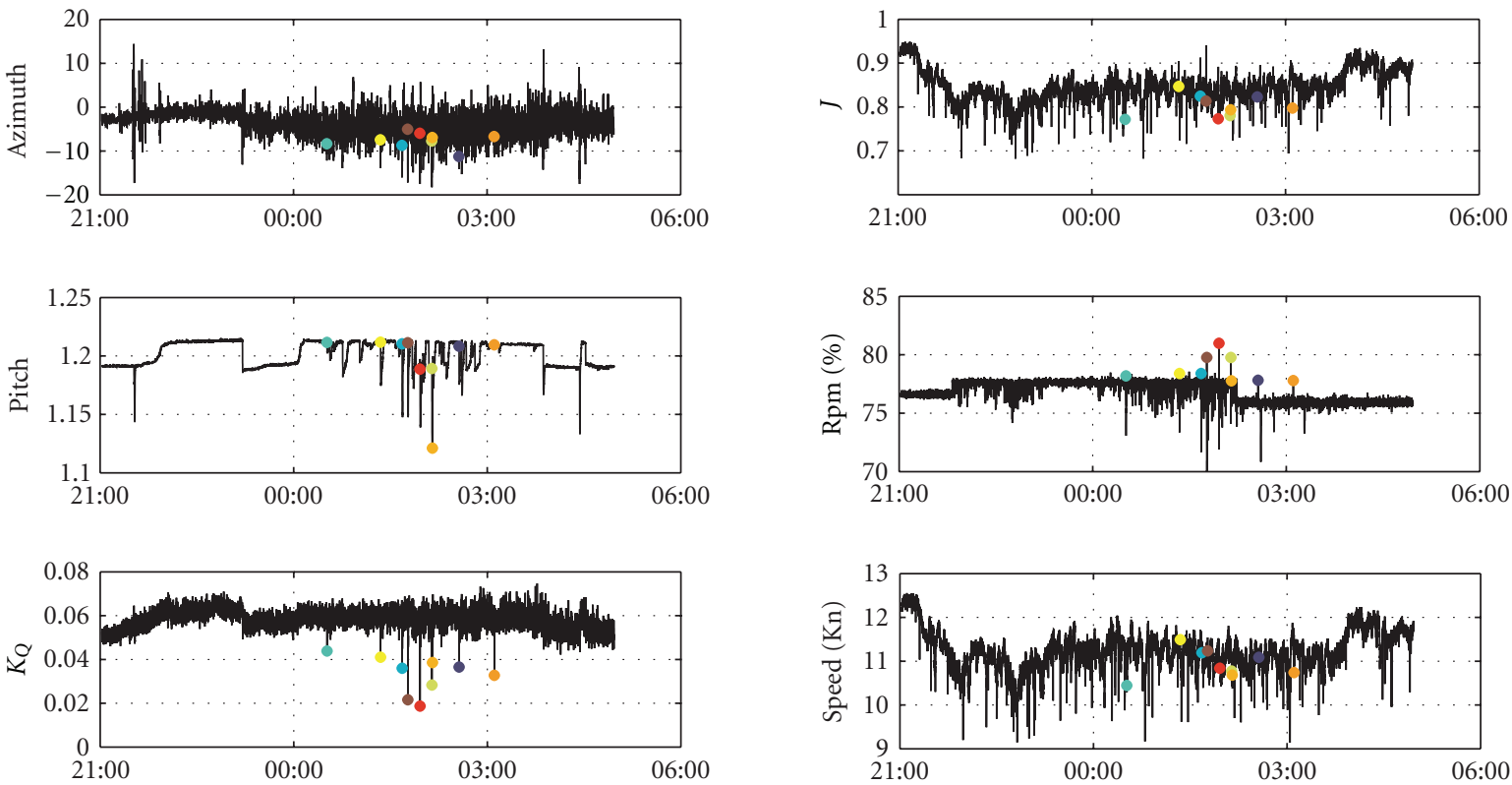

(a)
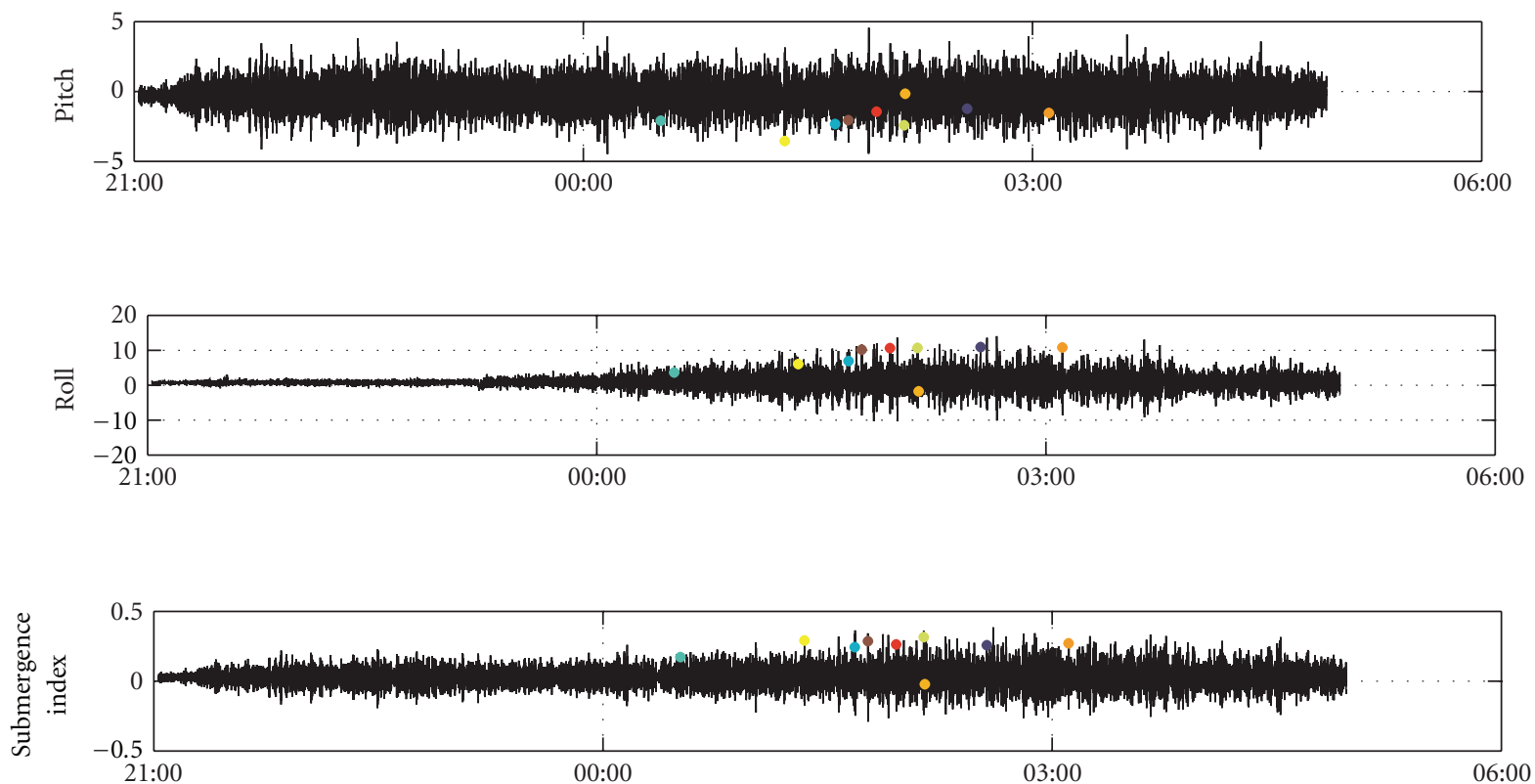

(b)

Figure 7: Time series showing HEMOS data (a) and ship environmental data (b) with the marked points.

the desired position, rather than aiming to a steady condition like in the transit case. The corresponding membership functions are reported in Figure 11. It has to be pointed out that during dynamic positioning the units are pointing inwards and hence the azimuth angle membership drops to zero. The resulting fuzzy set presents values which are well below the 0.85 threshold (Figure 12). The toolbox cannot be used during dynamic positioning, but at least it does not introduce false positives.

2.6. Test of the Toolbox. The data from the test ship are made available by Rolls Royce Marine in the form of time series covering one month. Therefore in this section the toolbox is adopted to scan through the data for ventilation events of a typical one month dataset. The ship is not operated continuously during one month, and trips to the platforms are alternated with time spent at the harbor. The noncontinuous operation of the ship is clear shown from both the propulsive system data and the ship motion presented in Figures 13 and 14 , respectively.

Although it is possible to analyze only the portion of the time series when the ship motions are large, the entire dataset is scanned with the toolbox. The resulting fuzzy set, with the point exceeding the 0.85 threshold marked by colored dots, is shown in Figure 15. 


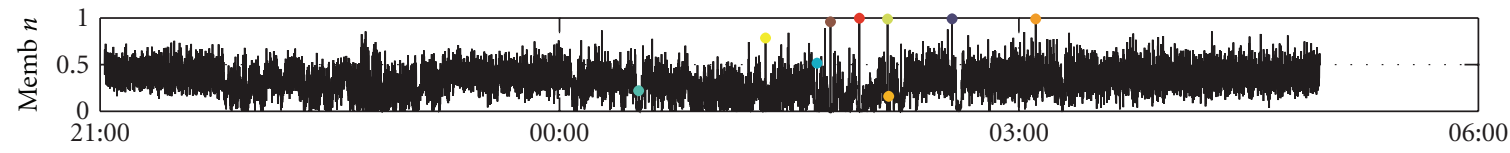

(a)

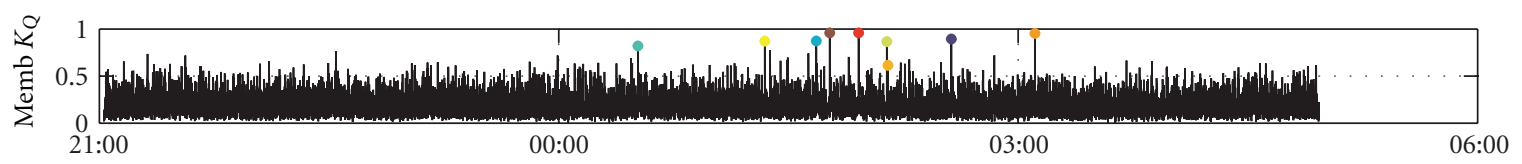

(b)

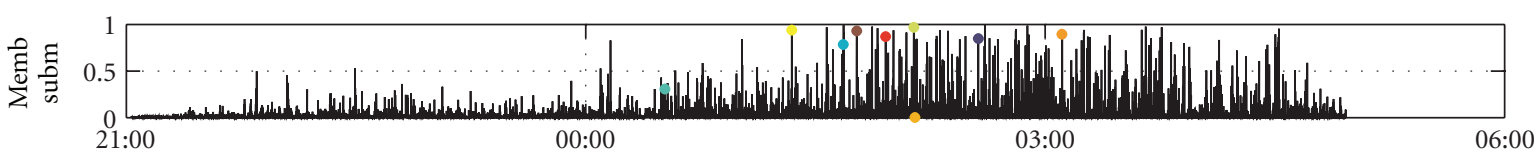

(c)

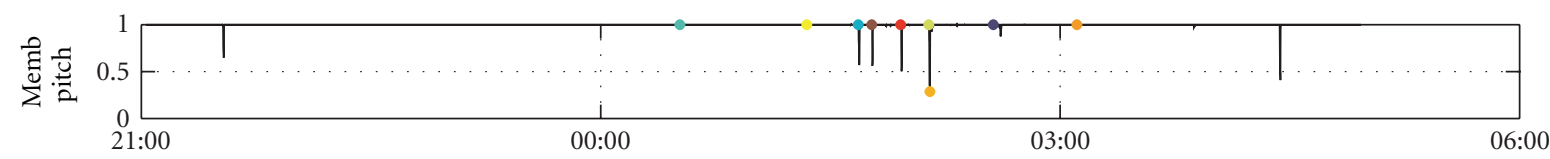

(d)

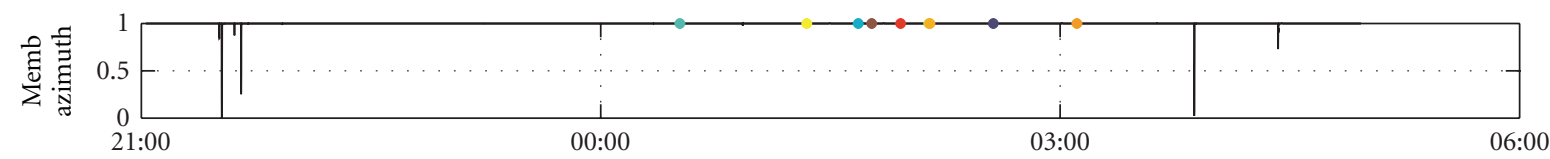

(e)

FIGURE 8: Calculated membership function for the analyzed time series, showing the marked points.

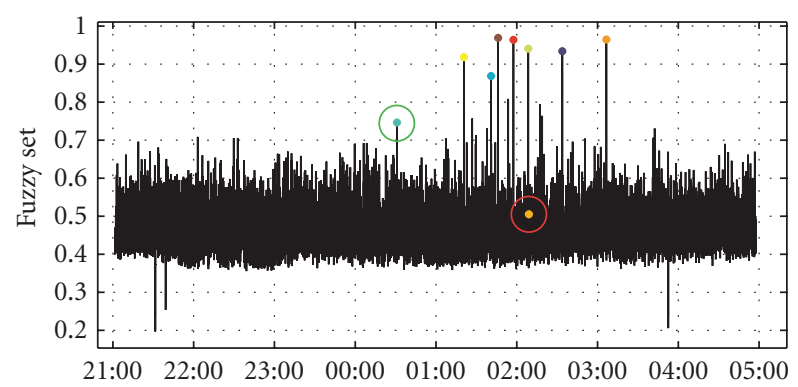

Figure 9: Time series of the Fuzzy set $F$.

Ventilation events have been identified in three different trips; those relative to second trip in which ventilation was identified are going to be further addressed. In Figure 16 the fuzzy set is plotted for the selected trip.

The identified events are also reported in the times series relative to the propulsive unit parameters and to the ship motions.

From both Figures 17 and 18 it is evident that the identified events occur in completely different ship operational modes. The first group was identified while the ship was in transit mode, whereas the second refers to a lower speed operation. The ship motions are also different; the last two events were recorded while the ship had a very limited roll motion. This marked difference is due to the different ship speed, but also to the fact that the ship changed course in between the two set of events as shown in Figure 19.

The toolbox proved to work also on a dataset different from the training one and to be able also to identify interesting events also in operational condition different from the transit.

\section{Application of the Toolbox}

The toolbox was applied to the data collected by both units installed on the test ship in the period January 2010June 2011. Data for the starboard unit are not usable for the month of January 2010, because the torque gauge was not fully operational. The analyzed period covers therefore almost one year and a half of ship operations. Using the fuzzy logic toolbox, 45 and 27 events have been identified for the port and the starboard units, respectively. It has to be remarked that 8 of the 45 events for the port unit were identified in the month of January 2010 when the other unit did not have usable data. Even without considering these 8 events, a difference of 10 events remains between the two units. This difference will be addressed in a specific section, while in this section the identified events are analyzed. The port unit is presented first. 


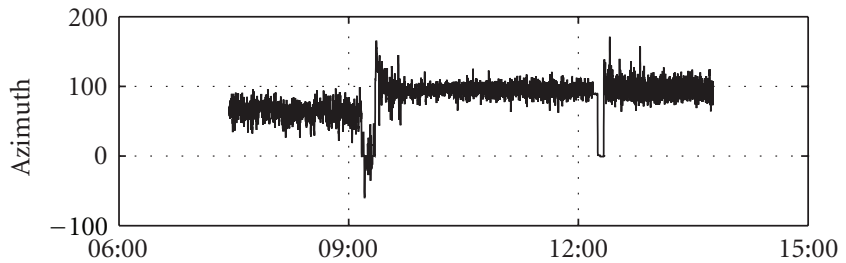

(a)

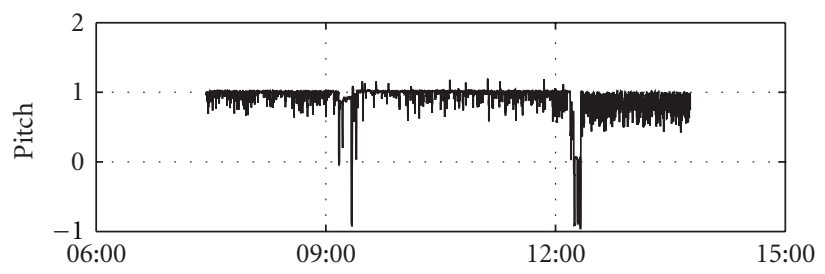

(c)

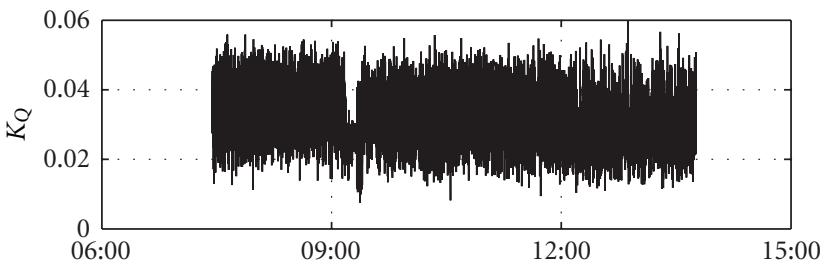

(e)

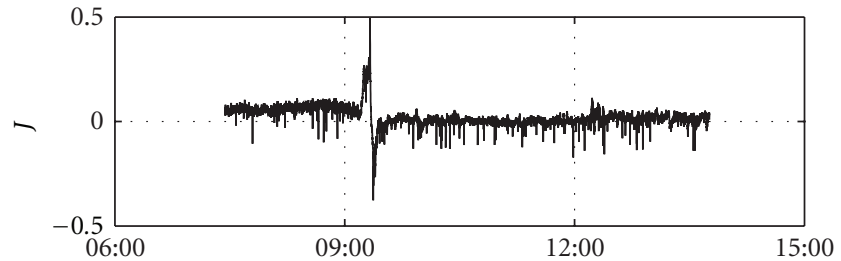

(b)

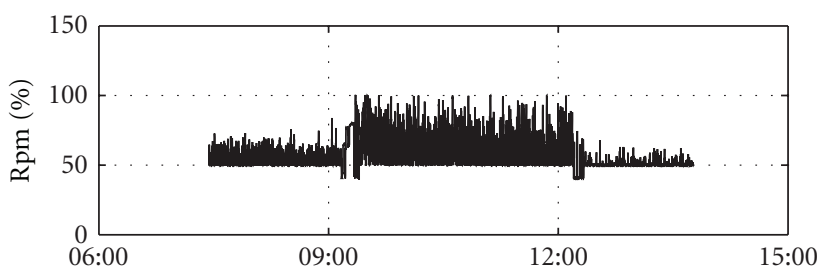

(d)

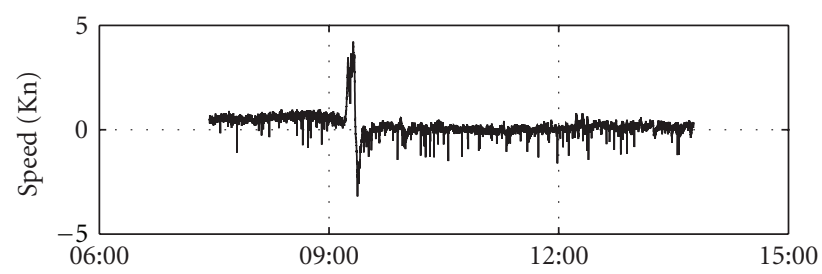

(f)

FIGURE 10: Time series of the propulsive system during dynamic positioning.

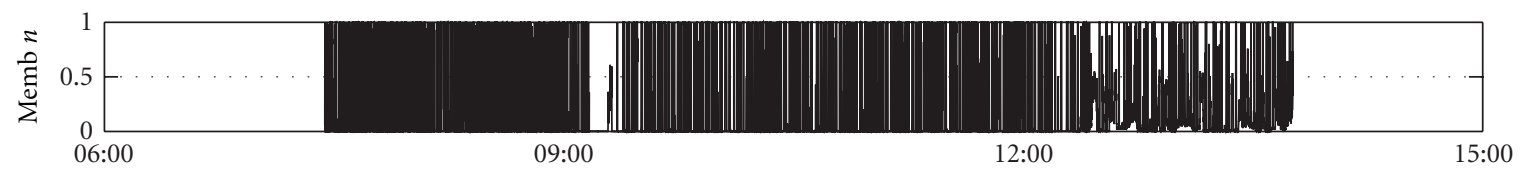

(a)

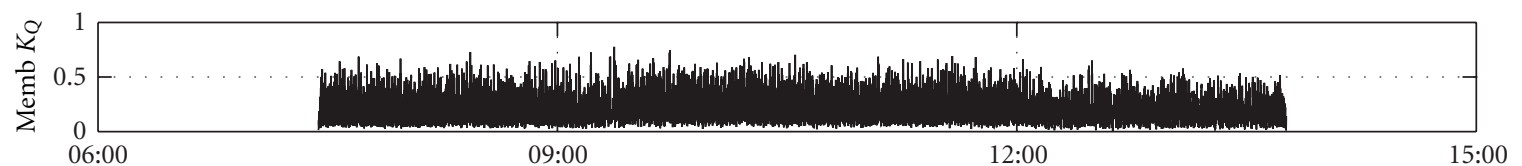

(b)

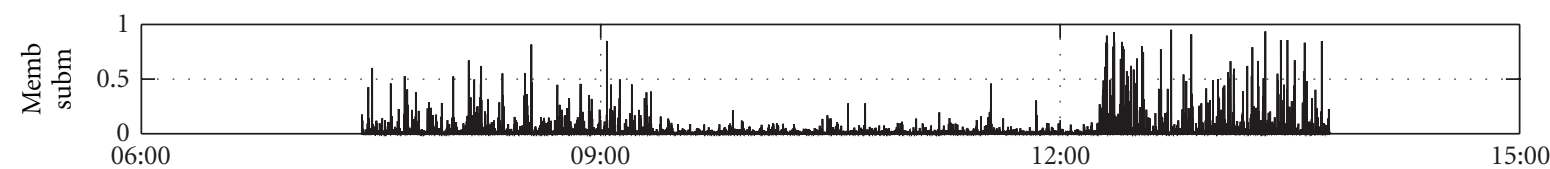

(c)

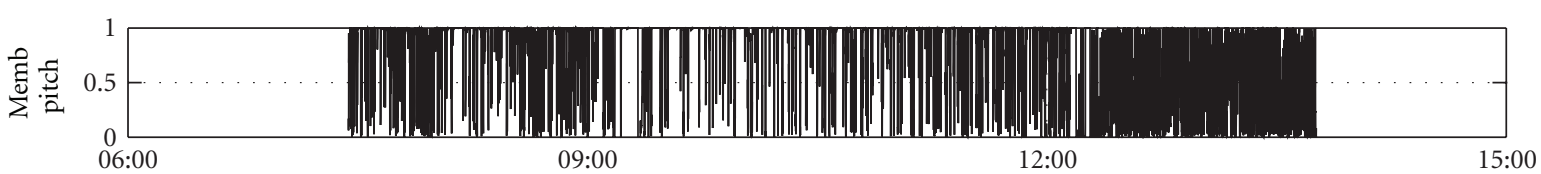

(d)

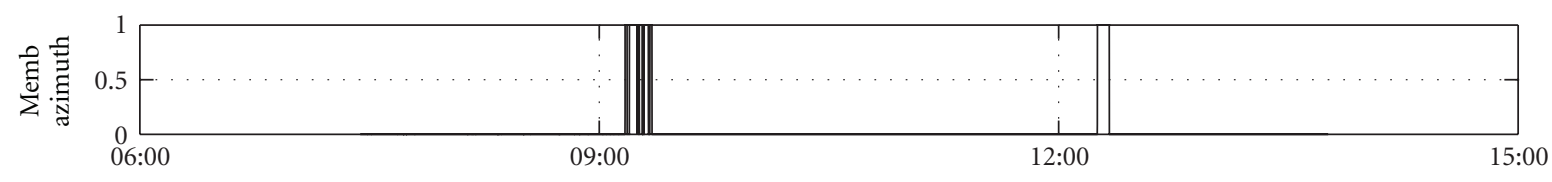

(e)

FIGURE 11: Membership functions during dynamic positioning operation. 


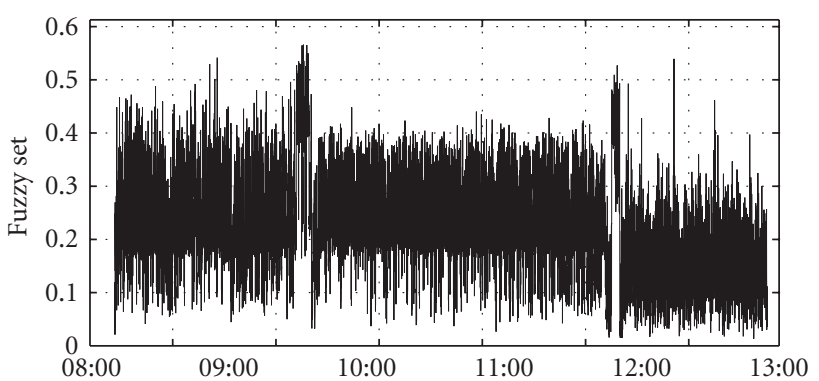

Figure 12: Fuzzy set $F$ for a typical dynamic position condition.

In Figure 20 the vessel speed when the events were collected is presented. The graph shows that most of the events occurred while the ship was in transit mode (ship speed around 10 Knots), although 4 events were recorded when the ship was operating at a lower speed. These last low speed events show that the toolbox is capable of detecting events also when the ship is not in transit condition, as it was in the training set. Most of the events were recorded when the azimuth angle was negative (Figure 21), which for the port thruster means that the unit was pointing outwards with respect to the ship centerline. However events with the unit pointing inwards were also detected.

The pitch and the rpm feedbacks are consistent with the assumption that most of the events occurred while the ship was in transit mode (Figure 22). Since the toolbox cannot handle the dynamic positioning condition, it follows that the events are mainly collected for the other most common ship operational mode, which is the transit.

The fact that the ship motions were large is not surprising. However it is interesting to notice that some events occurred when the ship did not have any significant roll angle but a large pitch angle (Figure 3 ).

The analysis of the starboard unit, in addition of the fact that fewer events were detected, shows some similarities with port unit, but also some relevant differences. The ship speed during the events is more scattered, showing more events at the lower speeds, although similarly to the other unit the transit mode seems to give more events (Figure 24).

The sign of the azimuth angle, being this the starboard unit, has a different meaning. In this case the unit is pointing outwards when the azimuth is positive.

If azimuth angles of the ports events are compared with the starboard ones, there are two main differences. The first difference is that the port unit shows events also for rather large inwards angles, whereas the starboard unit (Figure 25) does not show any events for large inwards angles. The second difference is in the magnitude of the angles. Although more events are available for the port unit, none of them shows an outwards angle larger than approximately 10 degrees.

The propeller pitch and rpm feedback graph (Figure 26) confirm that the starboard unit experienced more ventilation events in nontransit condition than the other unit.

The ship motion plot (Figure 27) does not show any particular difference with the port side. It has to be remarked

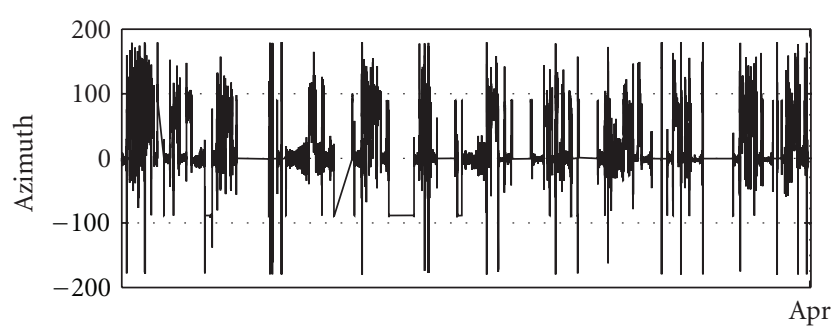

(a)

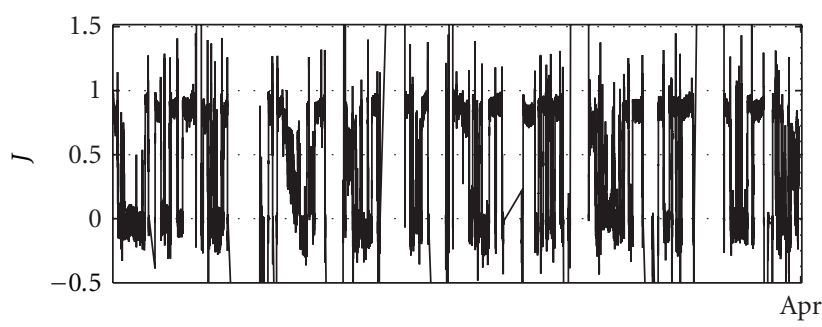

(b)

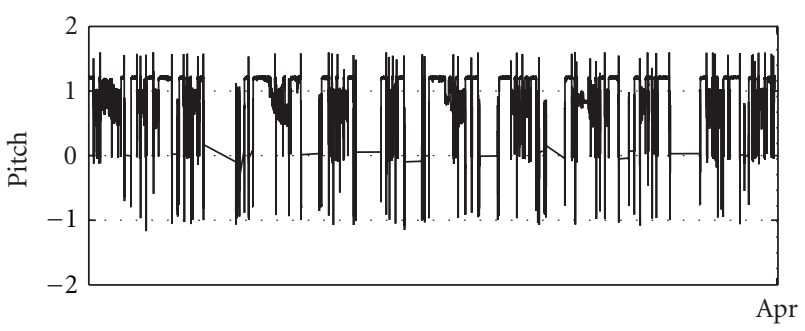

(c)

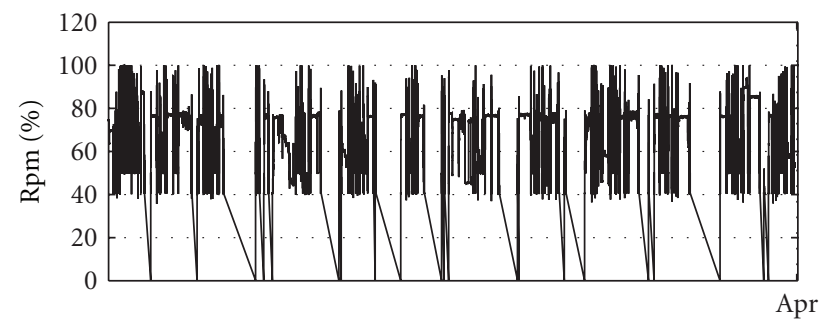

(d)

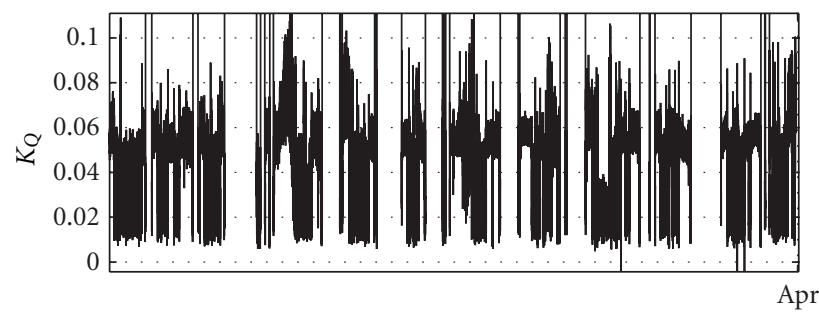

(e)

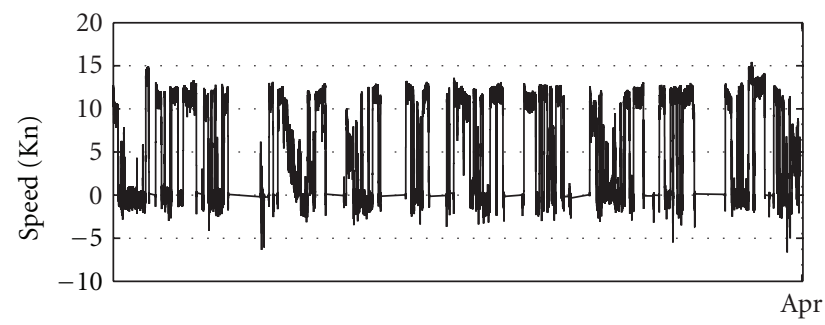

(f)

FIGURE 13: Propulsive parameters during the month of March 2011. 

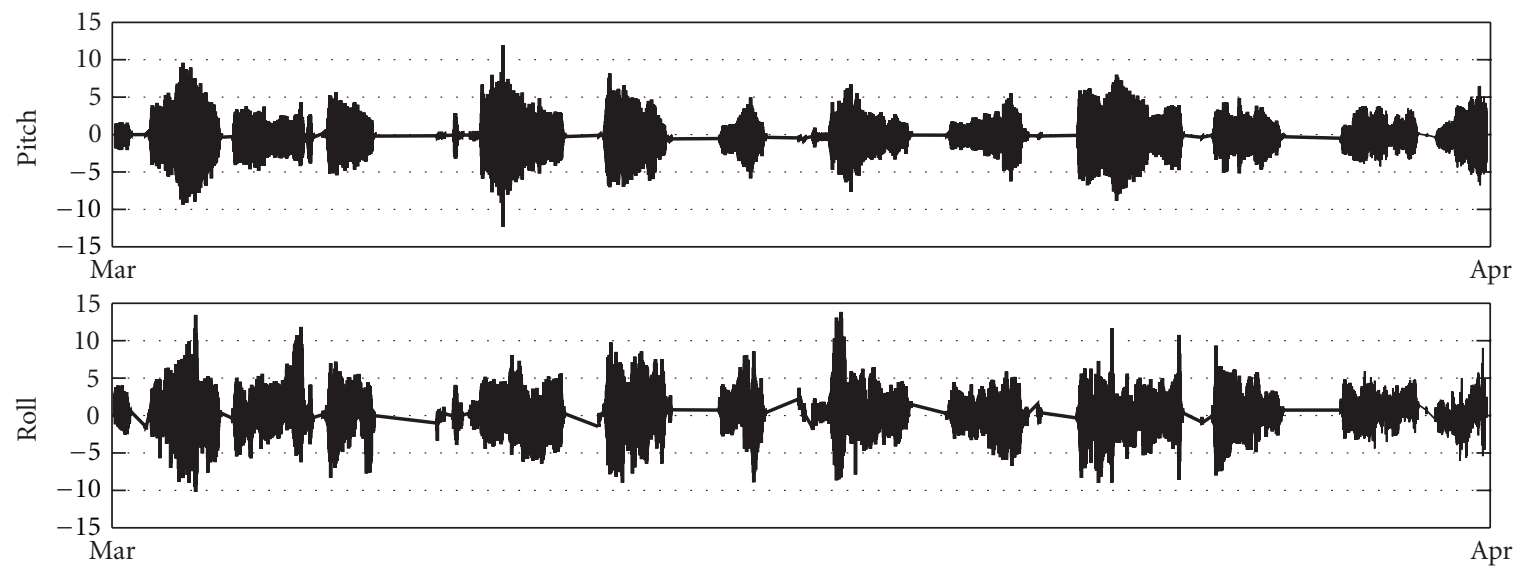

(a)

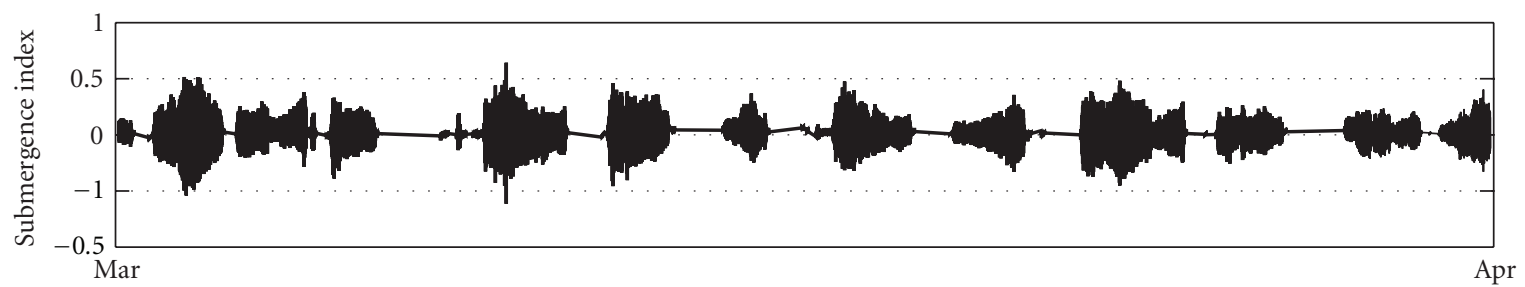

(b)

FIGURE 14: Ship motions during the analyzed period.

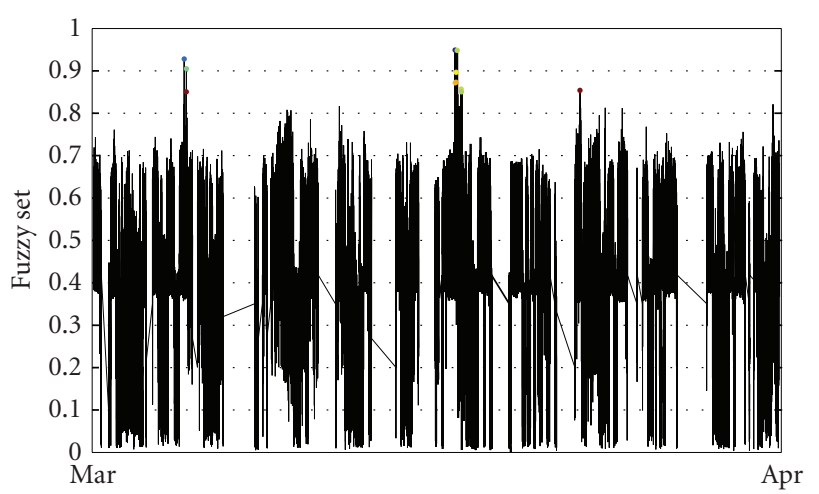

Figure 15: Fuzzy set for the month of March 2011.

that for the starboard unit negative roll angles lead to lower propeller submergences.

All the above-underlined differences led to the conclusion that there was some other parameter not taken into account that explained the discrepancies between the units. This idea was enforced when the cumulative sum of the events of the units was plotted against the number of months from the beginning of the analysis. The graph starts from the month of February 2010.

Figure 28 shows that the difference in the number of events collected up to June 2011 is probably due to the statistically short period of time considered. However

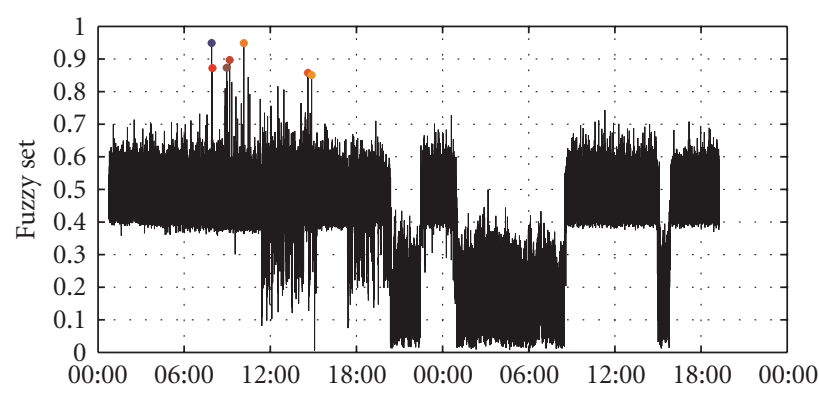

FIgURE 16: Time series of the membership function.

the statistics of the events collected so far show somewhat different trends.

3.1. Analysis of the Sea State during the Events. The test ship is a supply vessel which shuttles between platforms in the Norwegian Sea. The ship operates most of the time close to the Troll A platform. Figure 29 shows the track of the ship for the month of January 2010 and the position of the Troll A platform. This platform has a weather station and the collected data are freely available through the eKlima website, a service of the Norwegian Meteorological Service. Wave statistics are collected every 6 hours including wave spectral significant height, period, and direction. Assuming that the Troll A readings are representative of the sea state where the ship operates, it is possible to have a rough estimate of the wave statistics the ship was experiencing during the events. 


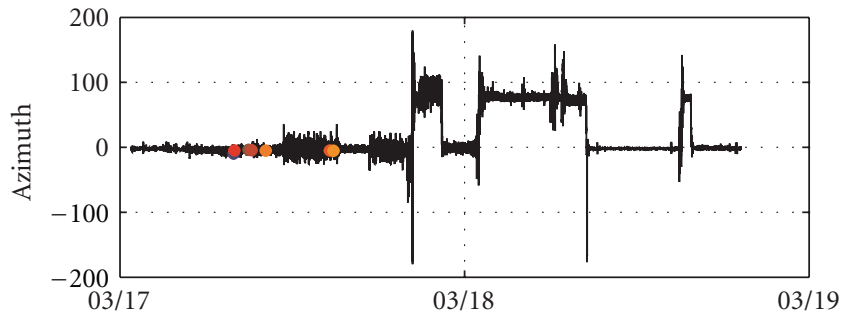

(a)

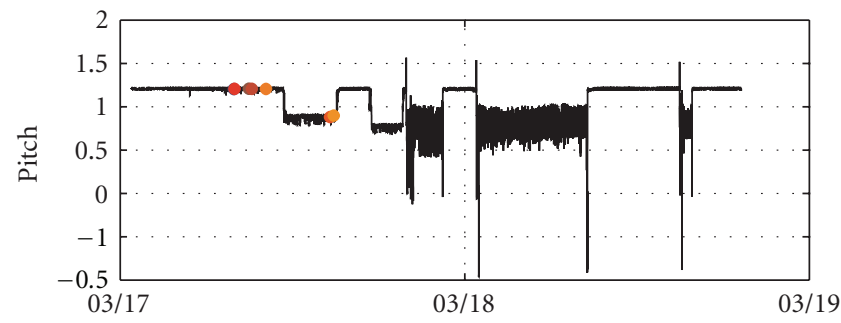

(c)

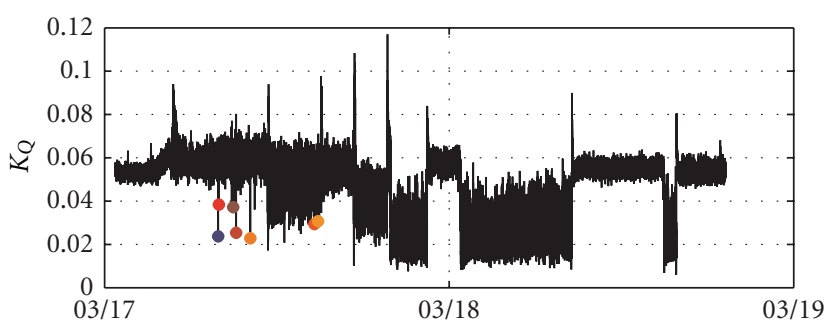

(e)

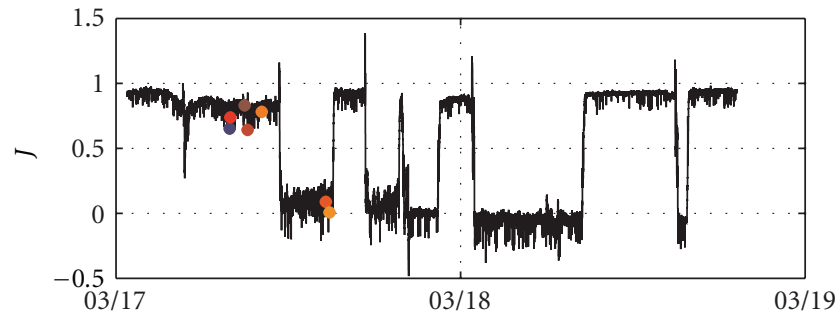

(b)

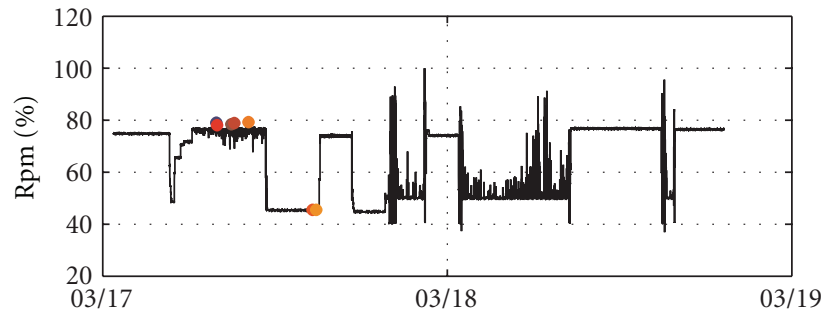

(d)

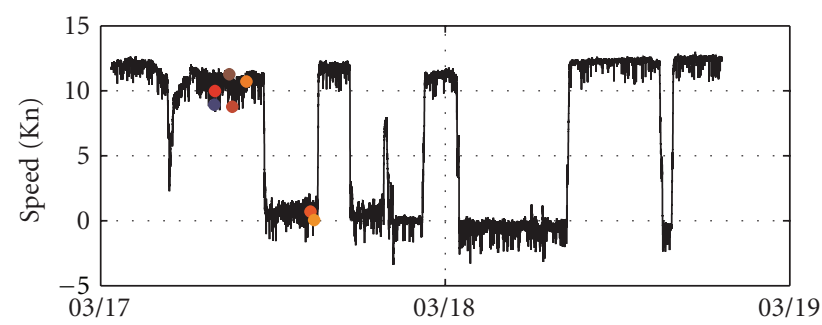

(f)

FIGURE 17: Propulsive system parameters during the analyzed period.

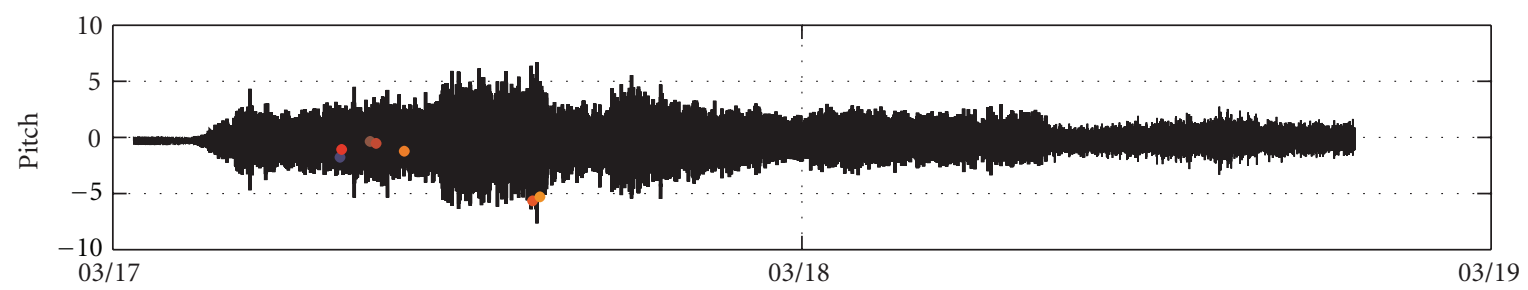

(a)

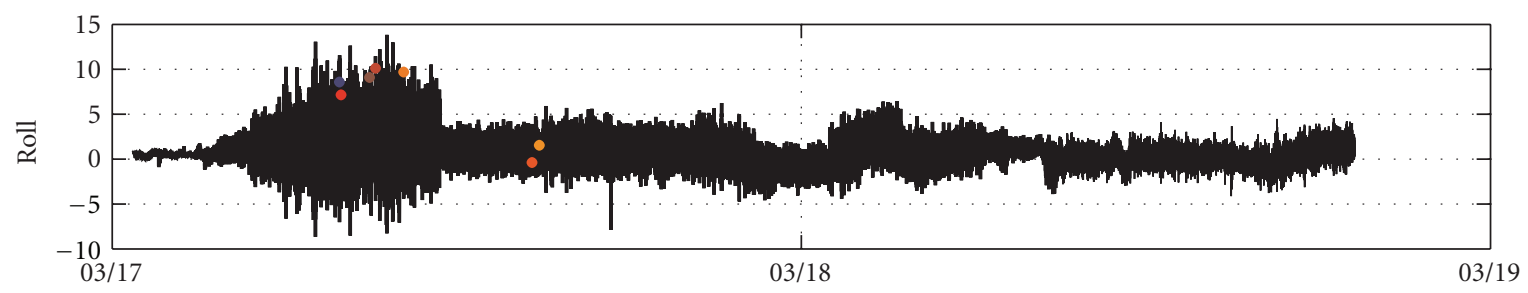

(b)

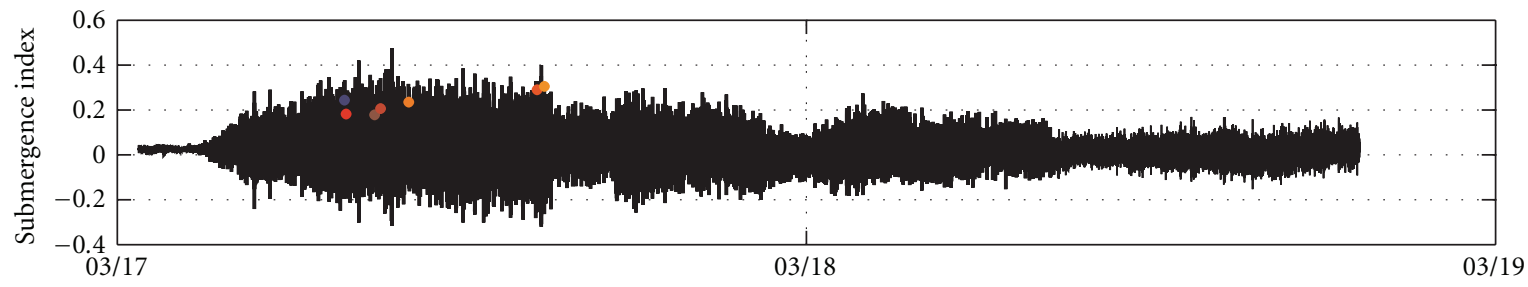

(c)

FIGURE 18: Ship motions during the analyzed period. 


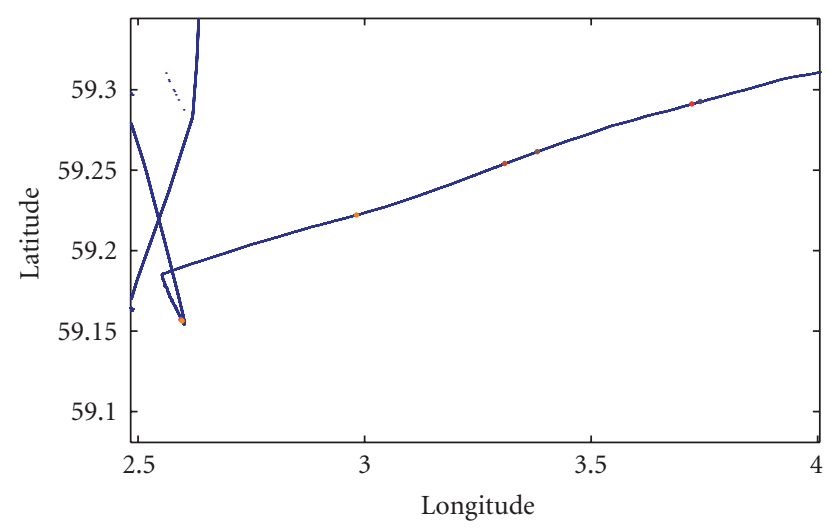

- Ship track

Figure 19: Part of the track of the analyzed trip.

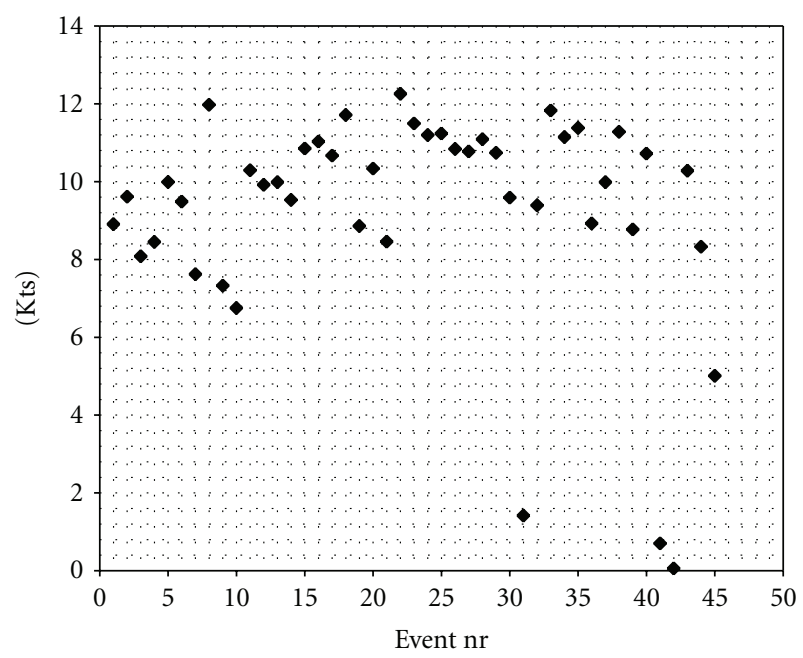

- Vessel_Speed'

FIGURE 20: Vessel speed during the events-port unit.

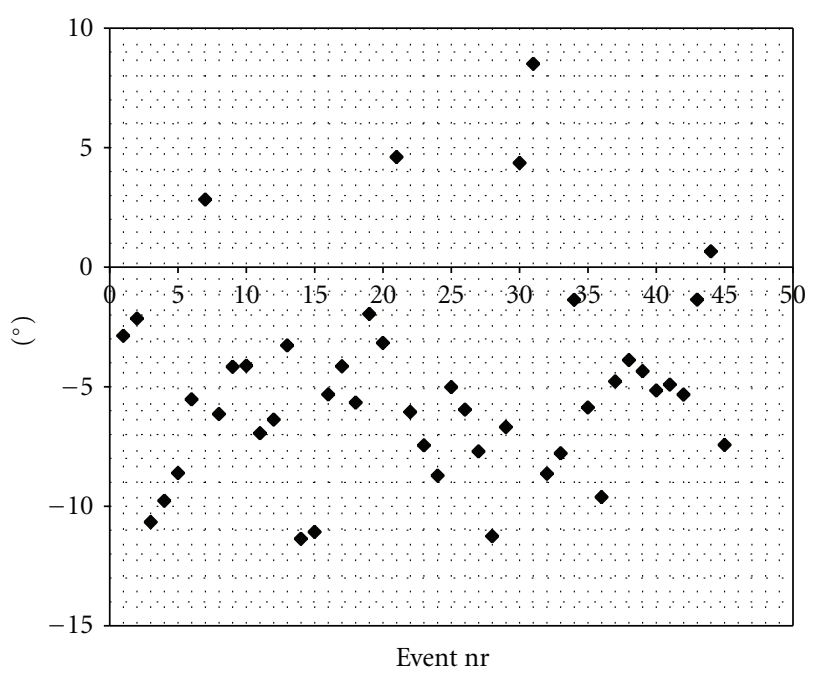

- Azimuth_Feedback'

FIGURE 21: Unit azimuth angle during the events-port unit.

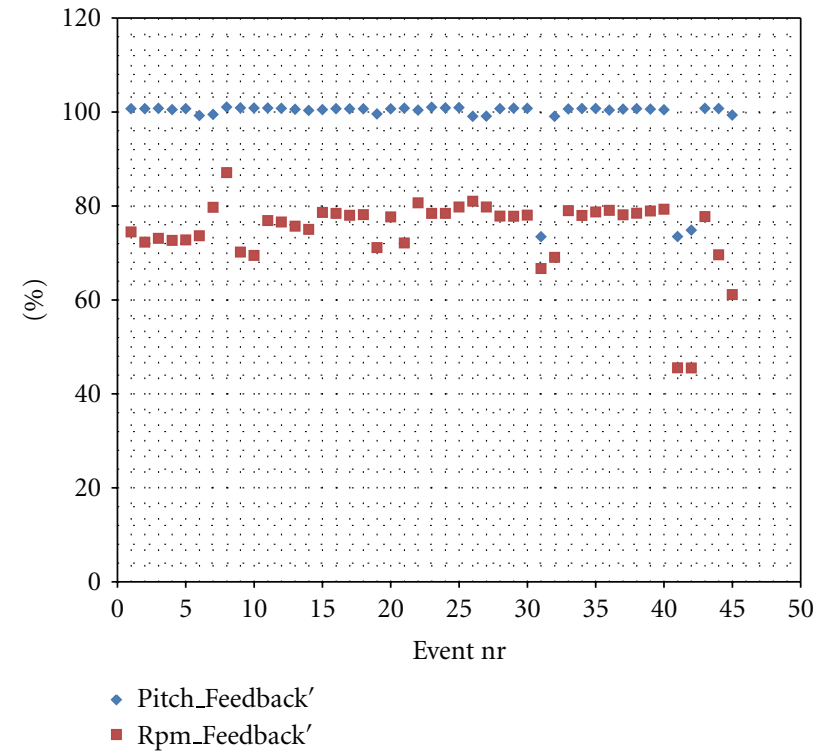

Figure 22: Propeller pitch and rpm feedback during the eventsport unit.

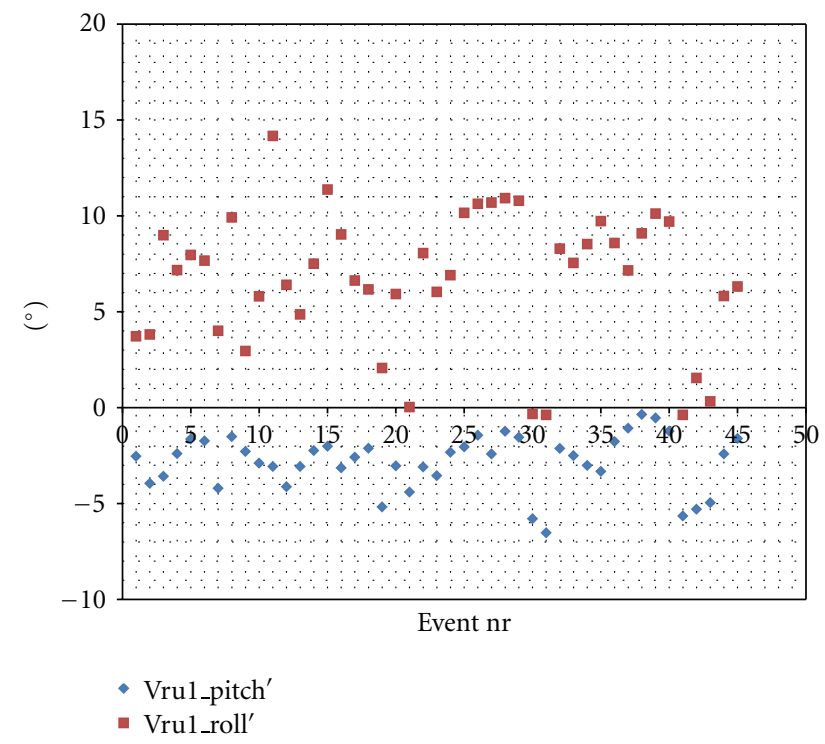

Figure 23: Ship Motions during the events-port unit.

In Figure 30 the wave first peak period is plotted against the wave significant spectral height for the identified events during the entire analyzed period of 18 months. By first peak period we refer to the largest peak in the wave spectrum. In the figure the blue and the red dots refer to the port and the starboard unit, respectively. The predominant wave first peak period during the events is around 8.5 seconds, which is a fairly common wave period for the Norwegian Sea. The significant height ranges from 2 meters up to 5.5 meters, with a concentration around 4 meters. One possible interpretation of the plot is outlined here. The lack of events at wave 


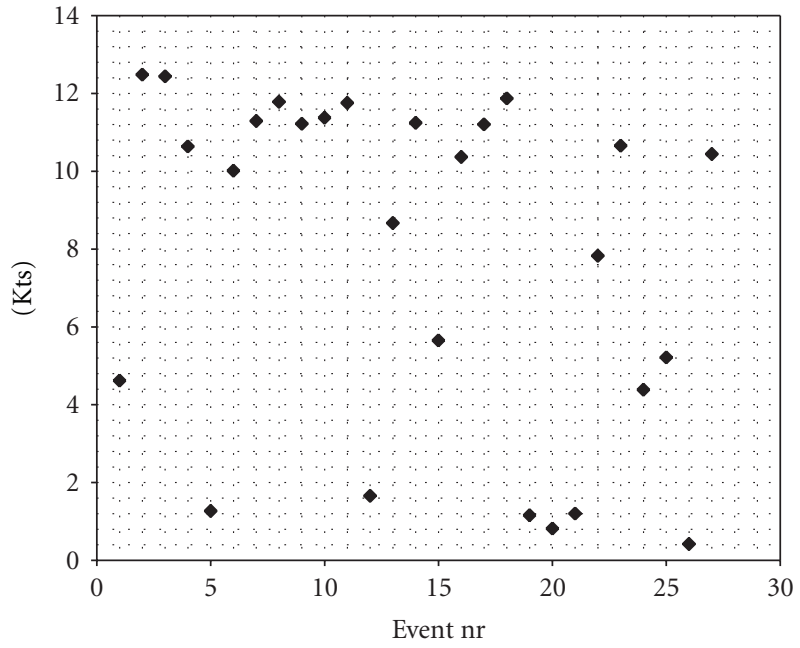

- Vessel_Speed

FIGURE 24: Vessel speed during the events—starboard unit.

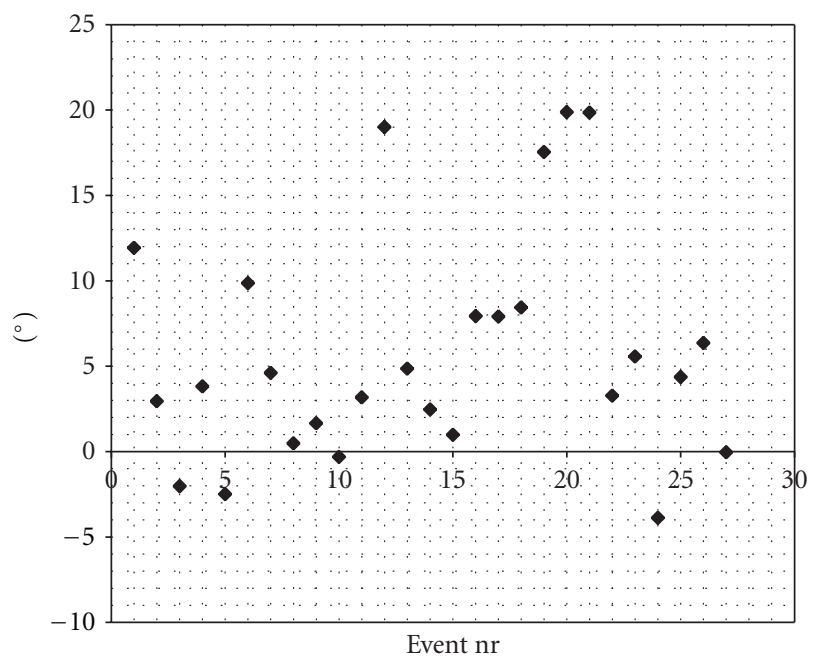

- Azimuth_Feedback'

FIGURE 25: Unit azimuth angle during the events—starboard unit.

significant height larger than 5.5 meters is probably due to the fact that the ship is not operated in rough sea states. The fact that fewer events have been identified at the smaller significant wave heights, although this is a more common sea state, is probably due to the fact that just the largest waves relative to this significant height might trigger a ventilation event. The number of events increases with the increasing significant height, up to the point where either the significant height is so large that it is infrequent or the ship is not operated in such large sea state. The last hypothesis is confirmed by comparing the distribution of relative frequency of occurrence of different wave heights at the Troll A platform for the entire period with the recordings when the ship was operating. These two distributions are presented in Figure 31 by a blue and a red curve, respectively. It seems

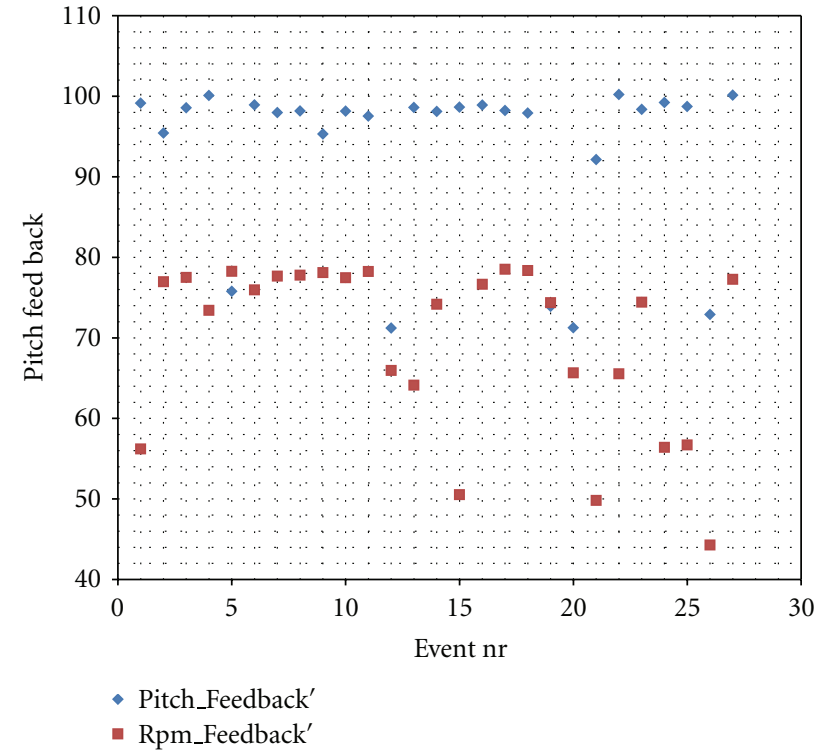

FIGURE 26: Propeller pitch and rpm feedback during the eventsstarboard unit.

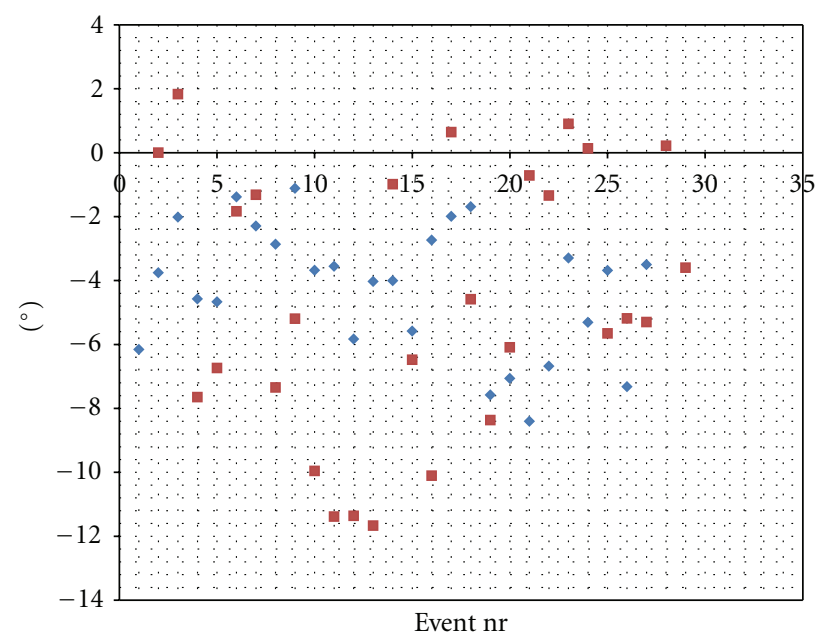

- Vru1_pitch'

- Vrul_roll'

Figure 27: Ship Motions during the events—starboard unit.

clear from the plot that the ship owner avoids operating the ship in heavy seas. The plot also confirms that the significant heights larger than 6 meters are rather infrequent at the Troll A location in the analyzed period.

In Figure 30 it can be noticed that also for the wave period distribution the two units show differences. The two distributions look very similar, but with the starboard unit slightly shifted towards longer periods.

This small statistical difference, combined with what already pointed out from the analysis of the time series, led to the idea that there was still something missing in the analysis. In other words the two units consistently show rather 


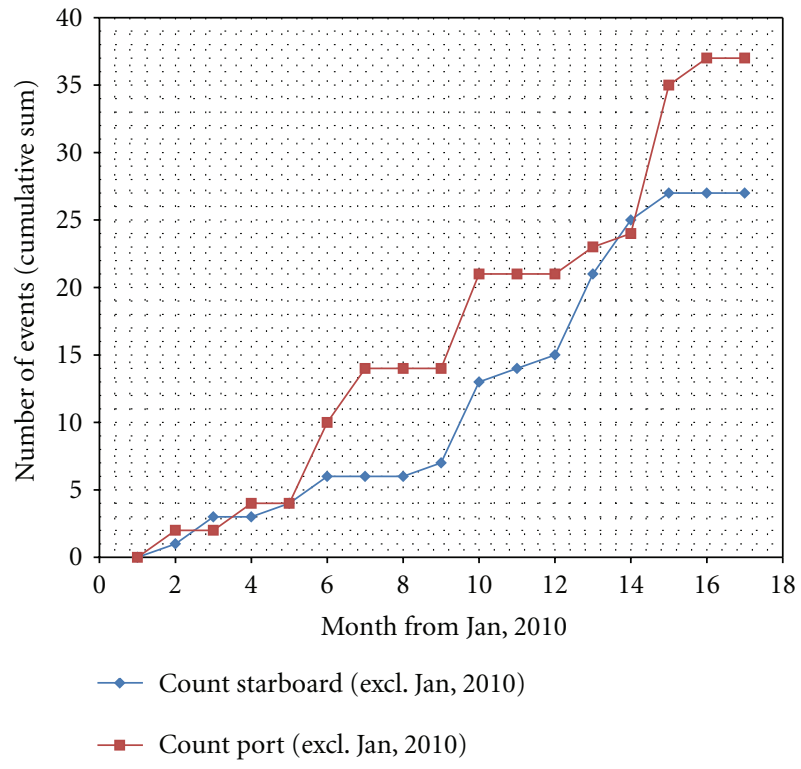

FIGURE 28: Cumulative sum of events for the starboard and the port unit.

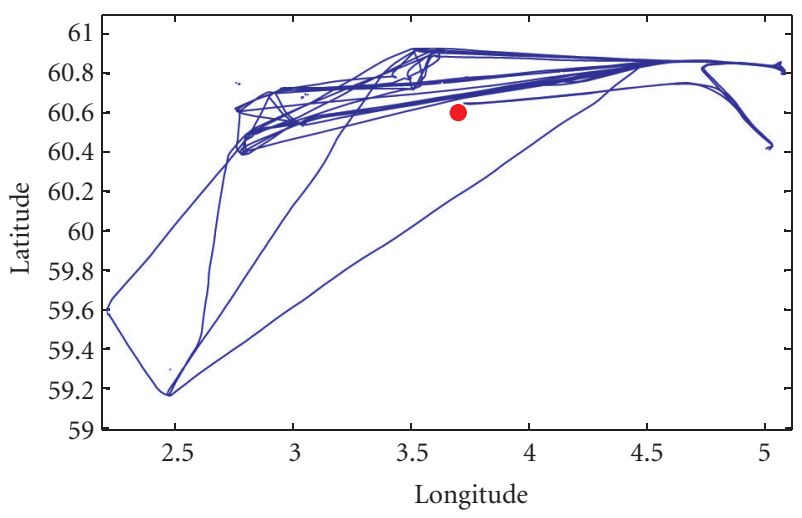

- Ship track

- Troll A platform

FIGURE 29: Ship tracks and Troll A platform position for the month of January 2010.

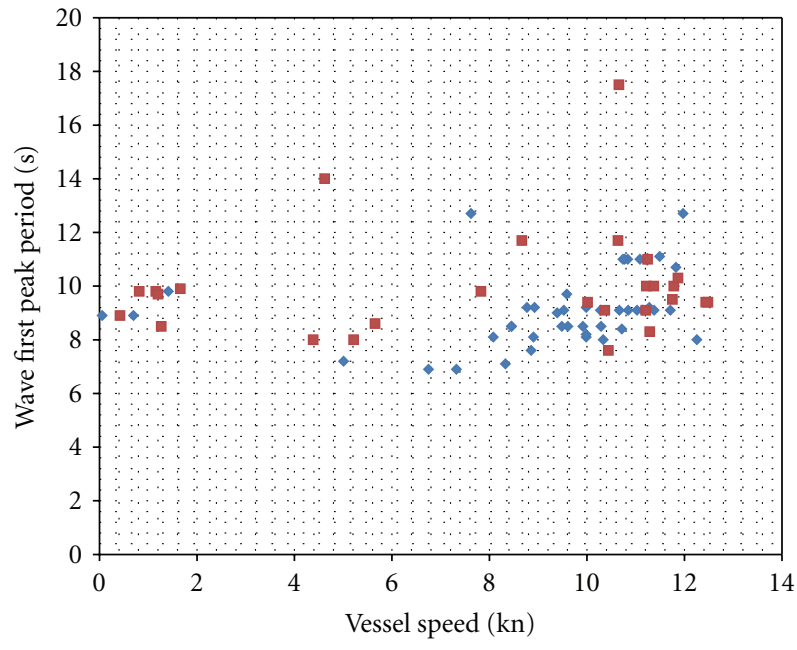

- TP-P

- TP-S

FIGURE 30: Wave first peak period versus wave significant spectral height during the events—blue for port and red for starboard. 


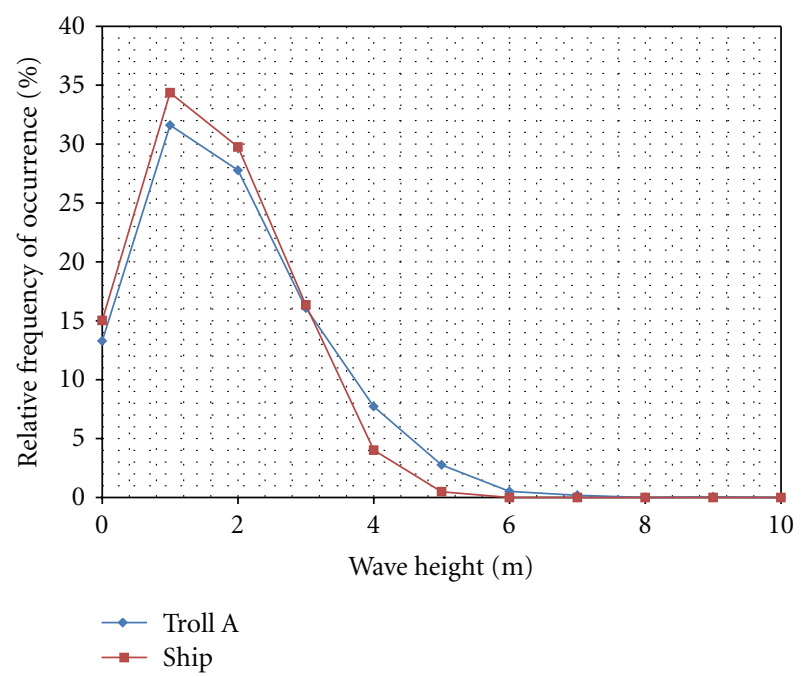

Figure 31: Comparison of Troll A and test ship wave significant height relative frequency of occurrence.

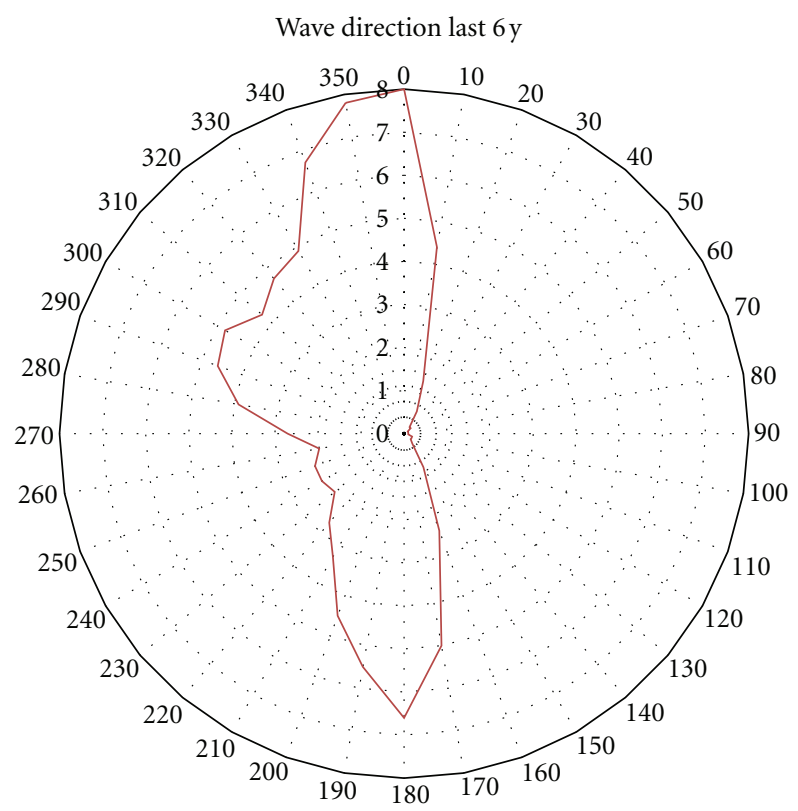

FIGURE 32: Relative frequency of occurrence of the wave direction 2004-2010 for the Troll A platform.

small, but noticeable differences which did not have a clear explanation in the parameters taken into account so far.

3.2. Estimation and Analysis of the Wave Encounter Angle. In order to explain the differences which have been pointed out in the previous paragraph, the wave encounter angle was added to the analysis. The wave encounter angle is defined as the angle between the wave direction and the ship course. It is zero when the ship course and the wave direction coincide (following seas), whereas it is 180 when two directions

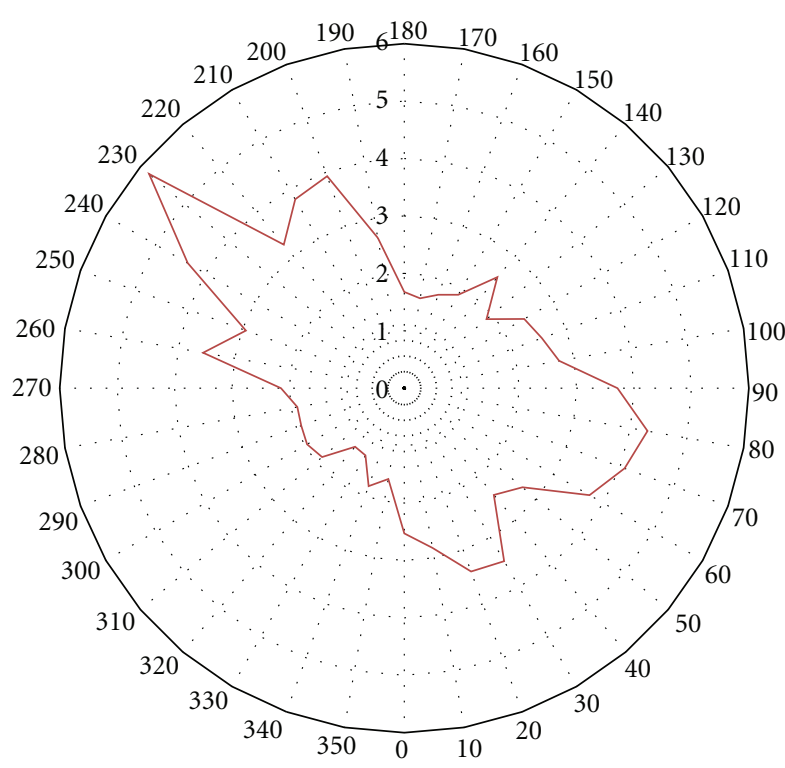

— Freq rel (Jan, 2010-May, 2011)

FIGURE 33: Relative frequency of occurrence of wave encounter angles.

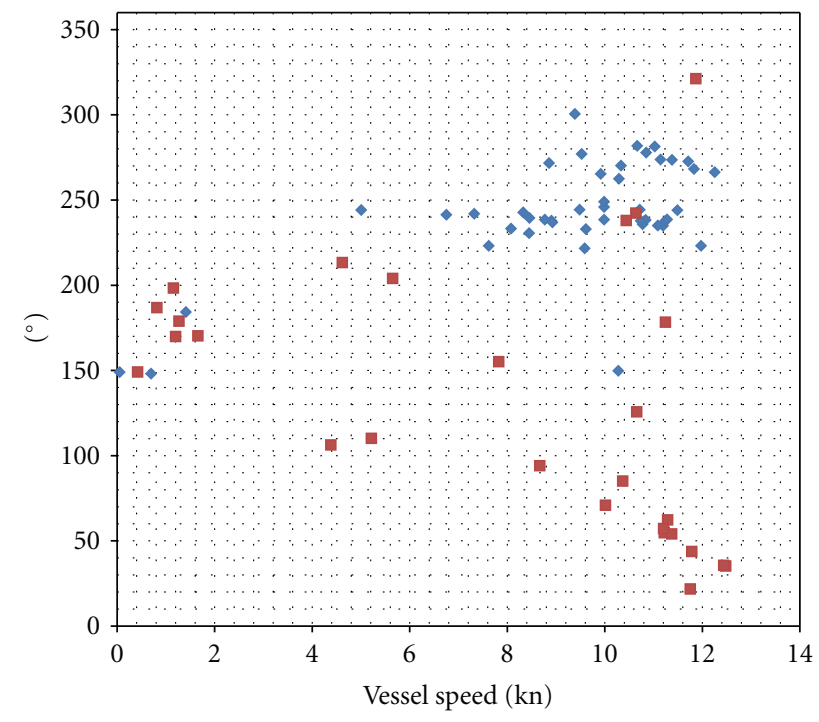

- Wave encounter angle-P

- Wave encounter angle-S

FIGURE 34: Wave encounter angle against ship speed-port unit (blue) and starboard unit (red).

are opposite (head seas). It is positive in counterclockwise direction, if the ship is seen from above.

If both the ship course and the wave direction have dominating values in the operation area, then it is likely that also the wave encounter angle of the ship will have some dominating directions. From Figure 29 it can be seen that the test ship steams prevalently on an east-west course. The Norwegian Sea, due to the presence of the mainland and 
due to the local atmospheric circulation, shows a relative frequency of occurrence of the wave direction (number of samples for a given wave direction divided by the total number of samples) which is not evenly distributed over the compass rose. This noneven distribution is shown in Figure 32, where the relative frequency of occurrence of the wave direction for the period 2004-2010 at the platform Troll A is reported (in the graph the atmospheric convention for the direction has been used and hence the direction represents the direction the waves are coming from).

Under the same assumptions used for the estimation of the wave height and period, it is possible to calculate the wave encounter angle for the relevant sea state and ship position. Once again the wave data available for the Troll A platform will be assumed to be representative of the wave data at the actual ship location. This assumption also means that, since the wave direction is updated just every 6 hours, the ship is supposed to experience the wave statistics of the observation closest in time. Therefore in the three hours preceding and the three hour following the observation the only parameter changing in the encounter angle calculation is the ship heading.

The calculated encounter angles are then divided in 10 degrees slots and their relative frequency of occurrence calculated. A filter is then applied to leave out of the statistics samples relative to significant heights smaller than 2 meters. The samples which have been acquired when the ship was sailing in sheltered areas have been left out as well. For this reason all the samples acquired east of $4.5^{\circ}$ in longitude, which is located approximately 15 miles offshore of the fjord where the ship is based, were not taken into account. Finally, only points with a ship speed greater than 2 knots have been taken into account, in order not to include dynamic positioning and low speed maneuvering. In other words the calculated relative frequency of occurrence represents the distribution of encounter angles of the waves which were deemed to potentially generate ventilation events while in transit mode.

The result of this analysis is presented in Figure 33. It is evident from the plot that the encounter angles are not evenly distributed over the 0-360 degrees range. This uneven distribution of the wave encounter angle might explain the differences in the events statistics, which were found from the analysis of the detected events. In particular the ship during the transit experiences most of the time port bow quartering seas and starboard stern quartering seas.

In Figure 34 the wave encounter angle during the events is plotted against the vessel speed for the port and the starboard unit. It has to be noted that the port unit is the windward one for encounter angles between 180 and 360 degrees, whereas the starboard one is the windward one for angles between 0 and 180. Not surprisingly the units experienced more ventilation events when they were the windward one, which in this case means that the ship was experiencing bow quartering seas during most of the port events and stern quartering seas during the starboard ones, consistently with the distribution in Figure 33. The differences in the ship response between bow quartering and stern quartering seas might be the cause for the differences between the two units in the event statistics that have been found so far. Figure 34 shows also that both bow and stern quartering seas can trigger ventilation on the windward unit. Ventilation on the leeward unit seems to be possible only during bow quartering seas. However this has to be checked against a larger number of events.

\section{Conclusions}

In this paper an analysis technique for the data collected by the HeMoS system is presented. The analysis adopts an ad-hoc developed fuzzy logic inference system toolbox, which proved to be useful in analyzing the data collected while the ship was in transit mode. Although the toolbox is not capable of handling the dynamic positioning mode, it proved to work also at low speeds, which was not expected in the development phase.

The toolbox was used to scan through the 18 months recording available in order to identify ventilation events. The toolbox identified a total number of 45 events for the port unit and 27 for the starboard unit. However 8 of the 45 events identified for the port unit were collected while the starboard unit acquisition system was not fully operational. It was shown that the different number of detected events between the two units seems to be due to the limited time span considered rather than to other causes.

The events were then analyzed from a statistical point of view with the aim of finding patterns in the events data. It was found that starboard unit shows a larger scatter in data when compared with the port unit. Including information about the sea state it was found that the majority of the events recorded for the port unit happened when the ship was experiencing port bow quartering seas, whereas the majority of the events for the starboard unit were recorded when the ship was experiencing stern quartering seas. This difference seems to be caused by the combination of the ship operation pattern and the dominating wave directions in the area of operation. However, more data is required to confirm this.

Although the time span covered in the present analysis seems rather long, the total number of events, considering both units, is just 72. Therefore the trends which have been outlined still miss a large number of samples to be confirmed and other might not be noted due to the lack of samples. It is then advisable to repeat this analysis when more data is available. It is demonstrated that in this kind of analysis it is important to include information about the sea state. In this case this was possible because the ship was operating in a stretch of sea where these data were available. However this is not the most common situation. It is advisable to try to develop methods to estimate the sea state from data which are more commonly available, such as the ship motions.

\section{Acknowledgments}

The authors would like to acknowledge Leif Vartdal and Leif Aarseth from Rolls Royce Marine for their support in the development of the fuzzy logic toolbox. The work is carried out as a part of the Era-Net Martec project PropSeas. 
The financial support from the Norwegian Research Council and Rolls-Royce Marine is appreciated.

\section{References}

[1] G. Kempf, "The influence of viscosity on thrust and torque of a propeller working near the surface," Transactions of the Institution of Naval Architects, pp. 321-326, 1934.

[2] H. Shiba, "Air drawing of marine propellers," Tech. Rep. 9, Transportation Technical Research Institute, 1953.

[3] F. Gutsche, "Einfluss der Tauchung auf Schub und Wirkungsgrad von Schiffspropellern," Shiffbauforschung, vol. 65, no. 6, pp. 256-277, 1967 (German).

[4] K. P. Fleischer, Untersuchungen über das Zusammenwirken von Schiff und Propeller bei teilgetauchten Propellern, Forschungszentrum des Deutschen Schifbaus, Hamburg, Germany, 1973.

[5] K. Koushan, "Dynamics of ventilated propeller blade loading on thrusters due to forced sinusoidal heave motion," in Proceedings of the 26th Symposium on Naval Hydrodynamics, Rome, Italy, September 2006.

[6] K. Koushan, S. J. B. Spence, and T. Hamstad, "Experimental investigation of the effect of waves and ventilation on thruster loadings," in Proceedings of the 1st International Symposium on Marine Propulsors (SMP '09), Trondheim, Norway, June 2009.

[7] A. Califano, Dynamic Loads on Marine Propellers Due to Intermittent Ventilation [Doctoral thesis], Norwegian University of Science and Technology, Trondheim, Norway, 2010.

[8] A. M. Kozlowska, S. Steen, and K. Koushan, "Classification of different type of propeller ventilation and ventilation mechanism," in Proceedings of the 1st International Symposium on Marine Propulsors (SMP '09), Trondheim, Norway, June 2009.

[9] L. A. Zadeh, "Fuzzy sets," Information and Control, vol. 8, no. 3, pp. 338-353, 1965.

[10] L. A. Zadeh, "Fuzzy logic and approximate reasoning," Synthese, vol. 30, no. 3-4, pp. 407-428, 1975.

[11] O. N. Smogeli and A. J. Sørensen, "Antispin thruster control for ships," IEEE Transactions on Control Systems Technology, vol. 17, no. 6, pp. 1362-1375, 2009. 

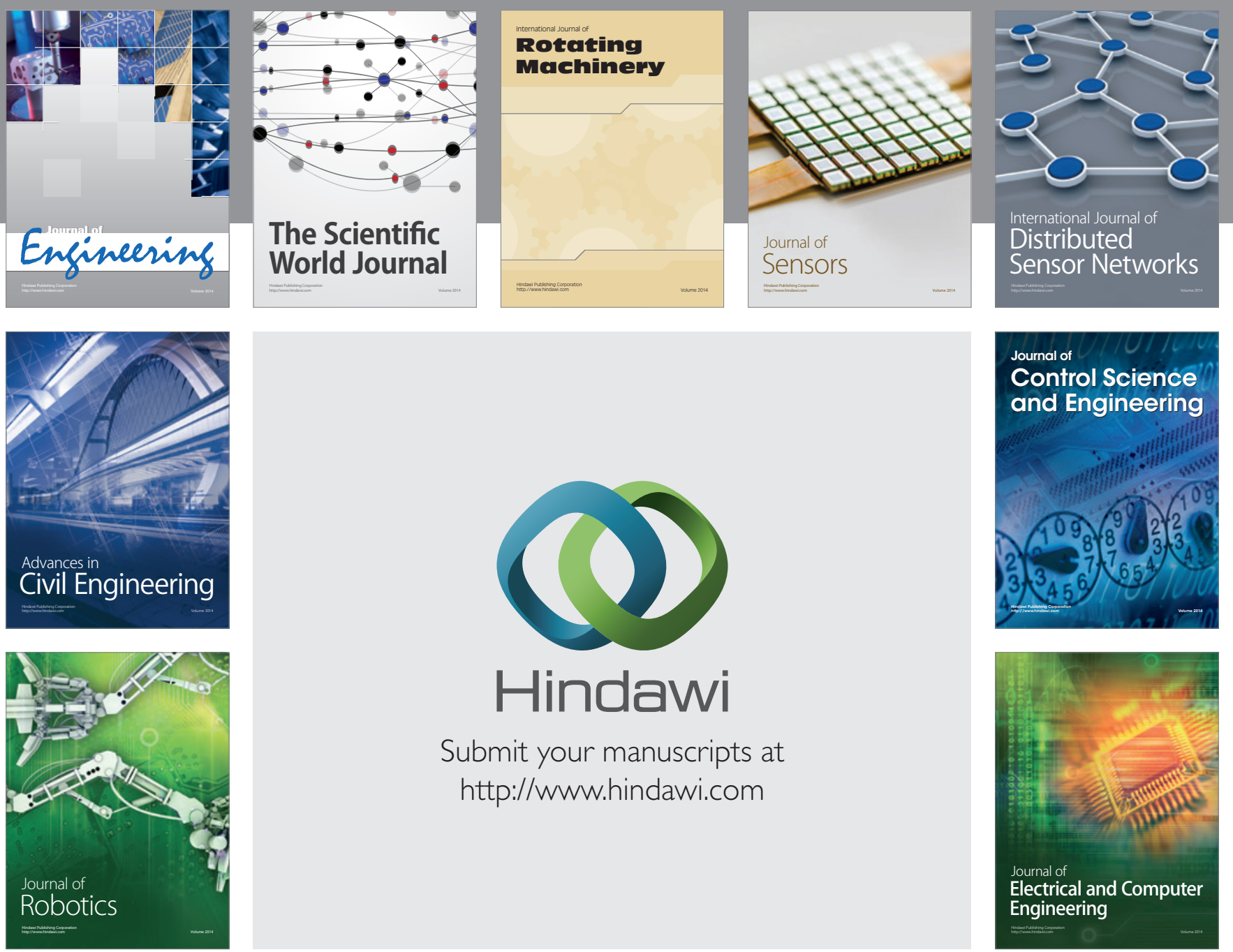

Submit your manuscripts at

http://www.hindawi.com
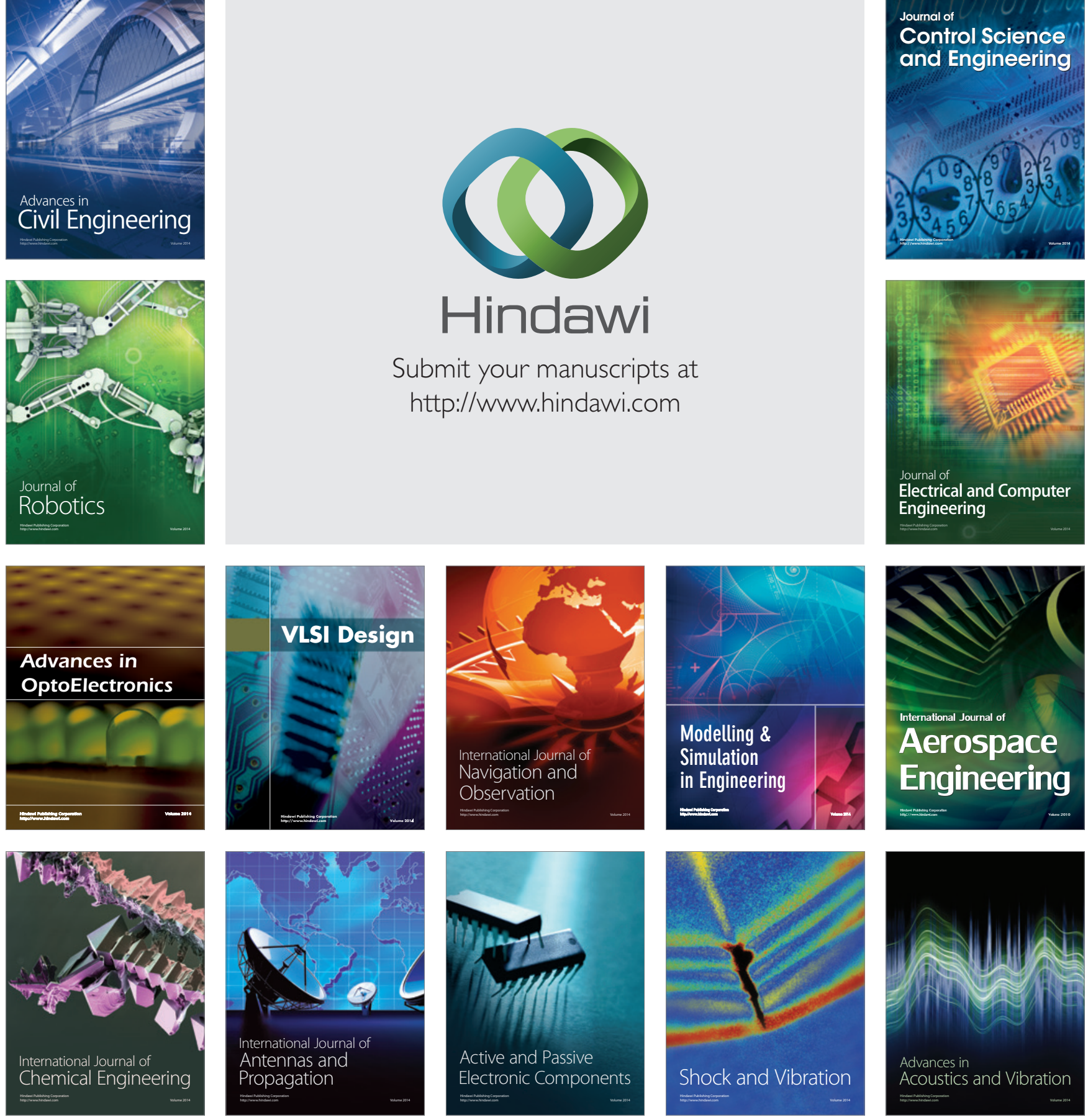